\title{
Dynamics of spontaneous spreading with evaporation on a deep fluid layer
}

\author{
Anne D. Dussaud and Sandra M. Troian \\ Department of Chemical Engineering, Princeton University, Princeton, New Jersey 08544-5263
}

(Received 14 April 1997; accepted 4 September 1997)

\begin{abstract}
The spontaneous spreading of a thin volatile film along the surface of a deep fluid layer of higher surface tension provides a rapid and efficient transport mechanism for many technological applications. This spreading process is used, for example, as the carrier mechanism in the casting of biological and organic Langmuir-Blodgett films. We have investigated the dynamics of spontaneously spreading volatile films of different vapor pressures and spreading coefficients advancing over the surface of a deep water support. Laser shadowgraphy was used to visualize the entire surface of the film from the droplet source to the leading edge. This noninvasive technique, which is highly sensitive to the film surface curvature, clearly displays the location of several moving fronts. In this work we focus mainly on the details of the leading edge. Previous studies of the spreading dynamics of nonvolatile, immiscible thin films on a deep liquid layer have shown that the leading edge advances in time as $t^{3 / 4}$ as predicted by laminar boundary layer theory. We have found that the leading edge of volatile, immiscible spreading films also advances as a power law in time, $t^{\alpha}$, where $\alpha \sim 1 / 2$. Differences in the liquid vapor pressure or the spreading coefficient seem only to affect the speed of advance but not the value of the spreading exponent, which suggests the presence of a universal scaling law. Sideview laser shadowgraphs depicting the subsurface motion in the water reveal the presence of a single stretched convective roll right beneath the leading edge of the spreading film. This fluid circulation, likely caused by evaporation and subsequent surface cooling of the rapidly spreading film, resembles a propagating Rayleigh-Bénard convective roll. We propose that this sublayer rotational flow provides the additional dissipation responsible for the reduced spreading exponent. (C) 1998 American Institute of Physics. [S1070-6631(98)00201-3]
\end{abstract}

\section{INTRODUCTION}

Many technological processes which take advantage of the rapid spreading of a thin liquid film along the surface of a deep liquid layer of higher surface tension could be controlled to better advantage if the mechanisms controlling the spreading rate were understood from a fundamental point of view. This is especially true of the spreading of volatile liquid films for which few studies have been reported, even though solvents are often used as the spreading vector in the preparation of organic or biological membranes. During the past two decades, studies of the spontaneous spreading of nonvolatile and immiscible thin films on a deep liquid support have attracted significant attention in the engineering community due to practical concerns with developing better antifoaming agents, liquid fuel fire extinguishers, and more accurate oil spill tracking algorithms. Spill tracking routines have been considerably improved by the incorporation of hydrodynamic models predicting the rate and extent of the contaminated region. Laminar boundary layer calculations have shown that during the late stages of an oil spill, the spreading rate is directly controlled by the balance between surface tension gradients generated at the air-liquid interface and the viscous drag generated in the water sublayer by the advancing oil film. ${ }^{1-9}$ Some of the more recent studies have generalized the spreading process to include different spreading geometries and film "feeding rates" as well as additional forces like gravity, capillarity, and surface diffusion. These modeling efforts have not yet been extended to include evaporation or dissolution, additional mechanisms which can significantly affect the spreading rate. Since most contami- nant liquids consist of volatile and/or miscible components, there remains a need to incorporate these effects into a realistic spreading model.

Another technological process which uses the rapid spreading kinetics just described is the formation of thin organic films for nonlinear optical coatings, microdevices, and biochemical sensors. The Langmuir-Blodgett process (LB) used to fabricate such films requires successive transfer of individual monolayers of organic molecules to form a thin solid film. ${ }^{10}$ A vertical substrate is successively immersed and withdrawn from a small water trough whose surface is covered by a compressed monolayer of the organic substance. The compressed monomolecular films are initially prepared by dissolving the organic compound in a volatile solvent like ethanol, hexane, or chloroform which spreads spontaneously and rapidly over the surface of a bulk water film. In order to form uniform monolayers, the solvent must transport the material homogeneously, evaporate completely from the water surface, and remain chemically inert. ${ }^{11}$ Though the LB process is an excellent technique for creating films of predetermined thickness and composition, it is fraught with technical difficulties associated with the deposition kinetics. A better understanding of the spreading kinetics in the presence of evaporation can help control or modify the waste inherent in producing nonuniform monolayers.

Spreading liquid films often contain surface active material like detergents, hydrocarbons, or phospholipids. These substances can significantly lower the surface tension of the liquid on which they are deposited and introduce gradients in surface concentration which in turn produce gradients in surface tension. Spontaneous flow toward regions of high sur- 
face tension, so called Marangoni flow, is typically a very rapid transport process whose speed is controlled by the spreading coefficient, $S$. Harkins ${ }^{12}$ defined this coefficient in terms of the tensions exerted at the junction of three phases to be $S=\gamma_{1}-\gamma_{2}-\gamma_{12}$ where $\gamma_{1}$ denotes the surface tension of the uncontaminated liquid (or solid) substrate, $\gamma_{2}$ the surface tension of the spreading liquid, and $\gamma_{12}$ the interfacial tension between the spreading and supporting liquid. This coefficient defines the force per unit length driving the spontaneous spreading of a liquid film over the surface of a liquid or solid support. For spreading films of molecular dimensions, the relevant spreading coefficient is $\Pi=\gamma_{1}-\gamma_{m}$ where $\gamma_{m}$ represents the surface tension of the monolayer covered liquid support. ${ }^{10}$

A simple force balance first proposed by Fay ${ }^{1}$ determines the temporal advance of a Marangoni driven thin film spreading on a deep liquid support. The analysis assumes a source of constant concentration which delivers a thin film whose interior is in plug flow. Fay reasoned that the force per unit length, $F_{S}$, driving the spreading process is the spreading coefficient, $S$, which derives from the Marangoni shear stress integrated over the length of the spreading film:

$$
F_{S}=\int_{0}^{L(t)} \frac{\partial \gamma}{\partial x} d x=S
$$

where $\partial \gamma / \partial x$ represents the local gradient in surface tension along the spreading film. This driving force is retarded by the viscous drag force per unit length, $F_{V}$, created by the viscous stress in the subsurface boundary layer integrated over the film length:

$$
F_{V}=\int_{0}^{L(t)} \mu\left(\frac{\partial u}{\partial z}\right)_{z=0} d x .
$$

The $x$ coordinate denotes the horizontal or radial direction of spreading and $z$ the vertical direction. The viscous stress at the surface can be estimated by $\mu U L / \delta$, where $U$ represents the surface spreading velocity which is order $L / t$. The viscous boundary layer thickness $\delta$ scales as $(\mu / \rho)^{1 / 2} t^{1 / 2}$ where $\mu$ and $\rho$ represent the subphase viscosity and density. From these estimates, the location of the leading edge is described by

$$
L(t)=K \frac{S^{1 / 2}}{(\mu \rho)^{1 / 4}} t^{3 / 4} .
$$

By tracking the receding edge of tracer particles sprinkled on the surface of water, experiments have confirmed the $3 / 4$ exponent as well as the coefficient controlling the speed of advance, $S^{1 / 2} /(\mu \rho)^{1 / 4}$ for nonvolatile and immiscible spreading films. ${ }^{3,7,13,14}$ Depending on the assumptions imposed on the spreading film, $K$ can range in magnitude from 0.665 to $1.52 .{ }^{13} \mathrm{~A}$ finite source of surface active material will produce a spreading exponent smaller than $3 / 4$. Scaling analysis for a finite source of spreading material produces $L(t)$ $\sim\left(A^{2} M^{2} t^{3} / \rho \mu\right)^{1 / 2(n+2)}$ where $n=0$ denotes a uniform front of infinite transverse extent, $n=1$ a rectilinear strip, and $n$ $=2$ an axisymmetric drop. ${ }^{9}$ The quantity $M$ represents the total mass of spreading material and $A=d \gamma / d \Gamma$, where $\Gamma$ represents the surface concentration. Our control experi- ments with nonvolatile liquids indicate that even when using microliter droplets to deposit the spreading film, the source behaves as an infinite reservoir during the first few seconds of spreading to reproduce the $3 / 4$ exponent. Only during the later stages of spreading does the finite volume become a rate limiting consideration, a regime beyond the scope of our present work.

The usual hydrodynamic description of Marangoni driven films on a deep liquid support assumes a constant spreading coefficient and no evaporation or dissolution. Davies and Rideal ${ }^{15}$ studied the spreading of acetone and ethanol at an air/water interface and measured a decreased spreading rate which they attributed to film depletion by desorption or evaporation. In studies of aqueous mixtures of anionic or cationic surfactants spreading on benzene, Joos and van Hunsel ${ }^{14}$ measured a spreading exponent of 0.575 . They speculated that the spreading coefficient should decrease in time since a finite mass of rapidly expanding film would suffer an increase in surface tension, $\gamma_{2}$, and interfacial tension, $\gamma_{12}$, through the dynamic dilution effect. Most recently, while investigating solitary wave behavior in turbulent spreading phenomena in the presence of dissolution, Santiago ${ }^{16,17}$ measured a spreading exponent of approximately $1 / 2$ for a highly soluble film of nitroethane spreading on a deep water support. In these limited studies, the presence of solubility or evaporation appears to decrease the spreading exponent though no systematic studies have been conducted to relate the mass loss directly to a reduced spreading coefficient or a reduced spreading exponent.

We have undertaken a series of experiments designed to elucidate the influence of evaporation on the spreading process. We present results on the spreading dynamics of several volatile and immiscible hydrocarbon films advancing over a quiescent water layer. To maximize the influence of evaporation on the spreading process, all experiments were performed in a completely unsaturated atmosphere. The vapor pressure and spreading coefficient of the different liquids used in the study were selected to provide reasonable evaporation rates and measurable spreading rates within the boundaries of our test cell. The visualization technique of choice was laser shadowgraphy since it allows noninvasive and precise tracking of the shape and speed of an advancing liquid front. This technique is superior to front tracking methods which require seeding the surface with talc or teflon particles which can unintentionally contaminate the water surface and/or disturb the more delicate features of the flow. Since shadowgraphy is very sensitive to regions of high curvature in free surface flows, it displays the entire shape of the spreading film from the point of deposition to the leading edge and can be used to monitor several fronts simultaneously.

Our studies with hydrocarbon films of different spreading coefficient and liquid vapor pressure reveal a common spreading exponent of approximately $1 / 2$, significantly smaller than the exponent for nonvolatile films. Differences in spreading coefficient or vapor pressure only affect the speed of advance but not the spreading exponent, which suggests the presence of a universal scaling behavior. As we discuss in later sections of this work, modeling the spreading 
TABLE I. Physical and interfacial properties of spreading liquids used.

\begin{tabular}{|c|c|c|c|c|c|c|c|c|}
\hline \multirow{2}{*}{$\begin{array}{l}\text { Spreading } \\
\text { liquid }\end{array}$} & \multirow{2}{*}{$\begin{array}{l}\text { Density } \\
\left(\mathrm{g} / \mathrm{cm}^{3}\right) \\
\left(25^{\circ} \mathrm{C}\right)\end{array}$} & \multirow{2}{*}{$\begin{array}{l}\text { Viscosity } \\
\quad(\mathrm{cp}) \\
\left(25^{\circ} \mathrm{C}\right)\end{array}$} & \multirow{2}{*}{$\begin{array}{c}\text { Boiling } \\
\text { point } \\
\left({ }^{\circ} \mathrm{C}\right)\end{array}$} & \multirow{2}{*}{$\begin{array}{c}\text { Vapor } \\
\text { pressure } \\
(\mathrm{mm} \mathrm{Hg}) \\
\left(25^{\circ} \mathrm{C}\right)\end{array}$} & \multicolumn{2}{|c|}{$\begin{array}{l}\text { Mutual solubility } \\
\text { with water } \\
\left(25^{\circ} \mathrm{C}\right)\end{array}$} & \multirow{2}{*}{$\begin{array}{l}\mathrm{S} \pm \\
\text { Pomerantz } \\
\text { et al. } \\
\text { (Ref. 18) }\end{array}$} & \multirow{2}{*}{$\begin{array}{l}\text { Our results } \\
\left(23^{\circ} \mathrm{C}\right)\end{array}$} \\
\hline & & & & & $\%$ w, solute & $\%$ w, water & & \\
\hline silicone oil & 0.965 & 965 & $\cdots$ & 0 & 0 & 0 & $\cdots$ & 9.4 \\
\hline toluene & 0.8622 & 0.5525 & 110.6 & 30 & 0.0515 & 0.0334 & $8.5\left(25^{\circ} \mathrm{C}\right)$ & 8.2 \\
\hline$p$-xylene & 0.8566 & 0.605 & 138.4 & 8.7 & 0.0156 & 0.0456 & $6.5\left(20^{\circ} \mathrm{C}\right)$ & 7.4 \\
\hline $\begin{array}{l}2,2,4 \\
\text { trimethyl } \\
\text { pentane }\end{array}$ & 0.6878 & 0.504 & 99.2 & 49 & 0.00024 & 0.0055 & $4.4\left(20^{\circ} \mathrm{C}\right)$ & 4.1 \\
\hline$n$-heptane & 0.6795 & 0.3967 & 98.4 & 45.7 & 0.00034 & 0.0091 & $1.3\left(20^{\circ} \mathrm{C}\right)$ & 2.3 \\
\hline
\end{tabular}

of a volatile immiscible film as a simple convective-diffusive problem, in which the evaporative mass loss is enhanced by the Marangoni driven spreading process but does not act on the film to reduce the spreading coefficient, shows that mass loss alone cannot change the 3/4 exponent observed for nonvolatile films. ${ }^{13}$ As some researchers have speculated, perhaps mass loss enhanced by evaporation can introduce some time dependence into the spreading coefficient thereby changing the temporal advance of the spreading film. In this paper we explore another possibility for the reduced exponent we have measured.

We present evidence of an unusual thermal boundary layer established in the supporting liquid. This thermal layer reflects the development of a vertical temperature gradient induced in the liquid support during the rapid spreading and evaporation process. We link the decrease in spreading exponent observed in volatile films to the presence of a Rayleigh-Bénard type convective roll which develops beneath the leading edge. This strong circulation pattern, only present in spreading volatile films, may provide the additional source of dissipation required to diminish the spreading exponent. We estimate the typical vertical temperature gradient required to establish this convective roll and find that it is easily achievable during the spreading process. In addition, the geometric characteristics of the convective roll bear resemblance to Rayleigh-Bénard cells created by purposely heating a liquid film from below. Unlike typical static studies, the observed roll propagates at the same speed as the leading edge within an imposed shear field established by the spreading film.

\section{EXPERIMENTAL DETAILS}

\section{A. Materials}

The model liquids used as received from the manufacturer were toluene $(99.8 \%$, Aldrich), $p$-xylene(99+, Aldrich), 2,2,4 trimethylpentane (99.9\%, Aldrich), and $n$-heptane (spectrophotometric grade, Mallinckrodt). The nonvolatile experimental control was silicone oil DC 200 of viscosity 10 poise (Fluka) which has been used in past studies of nonvolatile spreading films. The relevant material properties and spreading coefficients are shown in Table I. We have included two sources of measurement for the spreading coefficient, the eighth column lists the values collected from the literature by Pomerantz et al. ${ }^{18}$ while the ninth column lists our measurements. We measured surface tension with a platinum Wilhelmy plate and interfacial tension with a du Noüy platinum ring both of which were suspended from a Denver Instrument electrobalance with a resolution of $0.5 \mathrm{mg}$. All the water used in these studies was distilled and deionized ultrapure water $(18 \mathrm{M} \Omega \mathrm{cm})$ whose surface tension was measured to be $72.2 \pm 0.3 \mathrm{dyn} / \mathrm{cm}$ at $23{ }^{\circ} \mathrm{C}$. For the comparative studies in which we used talc powder to track the position of the leading edge, the talc (Aldrich) was purified before use by heating to $400{ }^{\circ} \mathrm{C}$ overnight.

\section{B. Visualization technique}

The experiments were performed in a circular glass dish of $16 \mathrm{~cm}$ diameter and $8 \mathrm{~cm}$ depth fit specifically with an optically flat bottom. The surface of the spreading film was visualized by laser shadowgraphy. A schematic of the experimental setup for viewing the free surface of the spreading film is shown in Fig. 1. Shadowgraphy is sensitive to small deflections of transmitted light caused either by variations in surface curvature for free surface films or by variations in refractive index for transmission through bulk fluids. We used this visualization technique in a vertical geometry for viewing the free surface of the spreading film and in a horizontal geometry for viewing the flow behavior in the water sublayer beneath the spreading film. The experimental assembly rests on a vibration-free optical table to minimize capillary waves from spurious mechanical vibrations in the environment. The glass cell is illuminated from below by a

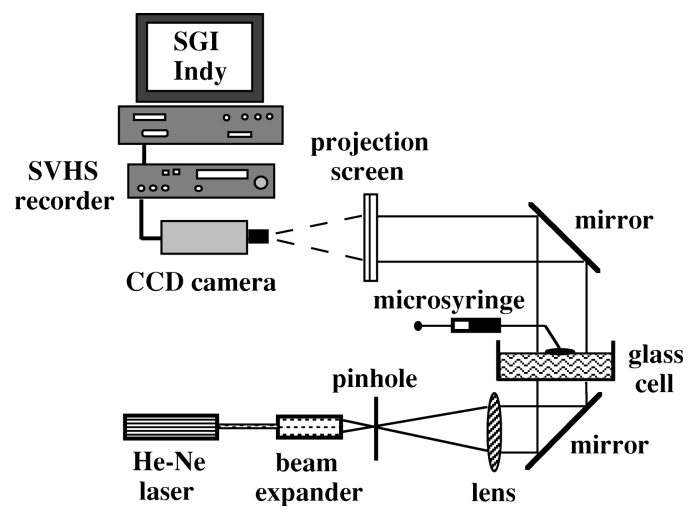

FIG. 1. Optical assembly for laser shadowgraphy in vertical geometry. 
beam of monochromatic light from a $1 \mathrm{~mW} \mathrm{He}-\mathrm{Ne}$ laser. The beam is expanded by first passing through a Newport spatial filter (Model 900) with a pinhole diameter of $25 \mu$ and then through a collimating lens (focal length $60 \mathrm{~cm}$ ) to produce a uniformly lit area of $9 \mathrm{~cm}$ diameter which illuminates a significant portion of the spreading film. The pinhole significantly increases image sharpness. The shadows created by the surface film curvature are projected onto a screen of ground glass and recorded by a high resolution Dage CCD camera (model MTI VE 1000) fitted with a Navitar TV zoom lens. These images are stored onto SVHS tapes and later analyzed using standard image processing software. ${ }^{19}$

The sideview experiments to visualize the subsurface flow behavior were performed in a square glass cell of dimensions $20 \times 20 \times 5 \mathrm{~cm}$. The flat glass sides, which were held together by a silicone adhesive sealant, minimized distortion of the light rays. The cell was illuminated from the side by a collimated light beam by modifying the lighting arrangement shown in Fig. 1. Parallel light enters the fluid from one side and is deflected by regions of the fluid which experience variations in the refractive index. Wherever the second derivative of the refractive index is nonzero, the light will be deflected from its original path to produce bright or dark regions ${ }^{20}$ on the projection screen depending on whether the light is brought to convergence or divergence by the local refractive index variation. Our studies reveal significant thermal variations and fluid circulation beneath the location of the leading edge which was well captured by this visualization technique.

\section{Experimental procedure}

All parts of the cell and drop delivery system which were in contact with the test liquids were carefully cleaned. The stainless steel needle and syringe plunger used to deposit the spreading droplet were degreased successively with acetone and methanol, then rinsed with pure water and dried. All glassware was first degreased by this same procedure, soaked in fresh sulfochromic acid for $10 \mathrm{~min}$, and finally rinsed with copious amounts of the ultrapure water. After this thorough cleaning the glass cell was stored in a clean glass box. Prior to an experiment, the delivery syringe was flushed several times with the test liquid of interest and then drawn to hold a specified amount in preparation for the actual droplet deposition onto the water surface.

The cell was filled with ultrapure water to a depth of 3 $\mathrm{cm}$. All experiments were conducted at a room temperature measured to be $23 \pm 1{ }^{\circ} \mathrm{C}$. During each experiment the cell remained opened to the atmosphere to provide completely unsaturated conditions in solvent vapor. The test area was kept as free as possible from dust and other environmental contaminants by large protective dividers housing the experimental assembly. The syringe delivery system, consisting of a $100 \mu \mathrm{l}$ precision Hamilton digital syringe, was capable of delivering small and precise volumes in the range of 2-4 $\mu \mathrm{l}$. We selected such small microvolumes of liquid in order to eliminate hydrostatic forces as an important initial spreading mechanism.

The syringe was mounted on a vertical position lift that allowed careful manual control of the liquid-filled needle.

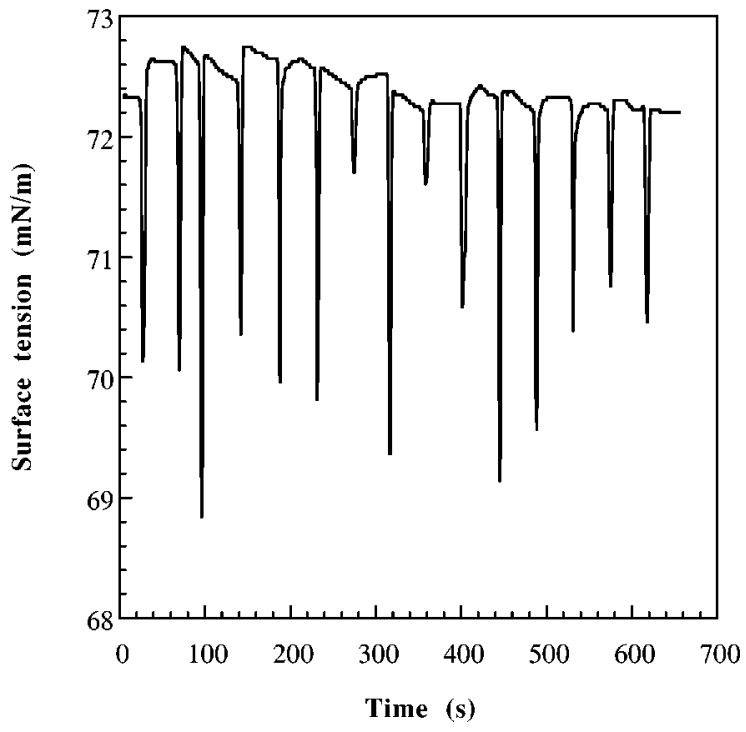

FIG. 2. Continuous in situ measurement of surface tension of toluene spreading on water. Measurements taken at a distance of $1.5 \mathrm{~cm}$ from point of deposition during successive depositions of toluene droplet $(\mathrm{V} \simeq 3 \mu l)$.

Before each run, the needle was positioned very close to the water surface such that once the apex of the droplet actually touched the water, the hydrocarbon was spontaneously drawn across the water surface by Marangoni stresses. This delivery system prevented the suspended droplet from being accidentally dropped or sprayed from any significant height which could introduce undesired inertial effects into the spreading process. Some residual liquid often remained in the needle tip presumably because the Marangoni and capillary forces responsible for pulling on the hydrocarbon droplet could not assume the entire volume. This residual volume presented no problem and was used to determine the volume of liquid deposited. Since the syringe piston was micrometer controlled, we manually adjusted the delivery system to add precisely the required volume for the next spreading event. The volume for each deposition event was therefore carefully controlled.

Cleaning the entire assembly after each spreading event was time consuming but necessary for the nonvolatile tests with silicone oil. We found a simpler solution for the volatile systems. A single droplet was deposited onto the calm water surface every $40 \mathrm{~s}$, a time period which we determined was sufficient for any surface film to evaporate and for the water surface to return to quiescent conditions. We determined that the surface tension of the air-water interface had returned to its original clean state after $40 \mathrm{~s}$ (for the volumes tested) by performing the following test. The surface tension of the air-liquid interface was measured in situ by using a Wilhelmy plate located at a distance of $1.5 \mathrm{~cm}$ from the needle tip. In Fig. 2 is shown the measurements during a series of successive depositions of toluene droplets. Within $10 \mathrm{~s}$ of each deposition, the surface tension of the water returned to a value of $72.2 \mathrm{dyn} / \mathrm{cm}$, indicating no surface contamination. A time period of $40 \mathrm{~s}$ between each deposition seemed to ensure that each spreading event was an independent and reproducible measurement. In order to collect statistically significant spreading measurements, we performed ten suc- 
cessive droplet depositions on the same water surface before thoroughly cleaning the cell as described above. We conducted 10-20 separate depositions for each volatile liquid.

\section{RESULTS}

\section{A. Surface film profiles of spreading films}

Typical surface shadowgraphs of spreading nonvolatile and volatile films are shown in Figs. 3(a), 3(b), 4(a), and 4(b). The black circular region at the farthest corners of the photographs delineates the maximum field of view allowed by the optical assembly. The outermost white band represents the highly curved leading edge of the spreading film. The rings of gray and black observed ahead of the spreading front, seen clearly in Fig. 4, represent capillary-gravity waves generated by the droplet placement onto the water surface. Since the speed of these waves was measured to be at least $24 \mathrm{~cm} / \mathrm{s}$, far in excess of the speeds of the hydrocarbon films described below, they are easily distinguishable from the leading edge of the contaminant film. The schematic diagrams in Figs. 3(c) and 4(c) trace the origin of the bright and dark bands in the shadowgraphs. The surface topology has been exaggerated for purposes of ray tracing. Concave regions of the film surface converge the incoming light to produce bright white bands while convex regions of the film diverge the light to produce dark gray or black regions on the projection screen. Regions of no curvature (either flat or constantly sloping areas) transmit the incoming light undisturbed to produce uniformly gray shadows. We note that the volatile films display much stronger curvature near the leading edge and near the source region. In the analysis described below, we monitored the advance of three distinct bands, labeled $L(t), B(t)$, and $R(t)$ in Figs. 3 and 4, when sufficient contrast allowed. We identify $L(t)$ with an elevated rim at the leading edge of the spreading film and $R(t)$ with the periphery of the droplet source. Though $L(t)$ is used to locate the position of the advancing rim, the measurements are actually taken at the trailing edge and not the forward edge of the white band. In the numerous shadowgraphs studied, the sharpest contrast for accurate image processing occurred at the trailing edge because of its proximity to the very dark region behind the rim. On the time scale of our measurements (approximately $2 \mathrm{~s}$ of spreading) the width of the white band did not grow in time so that $L(t)$ is identified with the leading rim of the contaminant film. The terms leading rim and leading edge will be used interchangeably.

We know with certainty that $L(t)$ accurately tracks the elevated rim at the leading edge of the spreading film by comparison with previous studies of nonvolatile films and with our own separate experiments using talc powder for visualization. For the silicone oil studies, our measurements of $L(t)$ reproduce previous measurements of the leading edge by surface seeding with teflon particles. ${ }^{3,7,14}$ For the volatile substances, we sprinkled pure talc powder onto the surface of an initially quiescent water surface. We monitored the receding talc front while illuminating the surface by laser shadowgraphy. Since the small talc particles blocked the
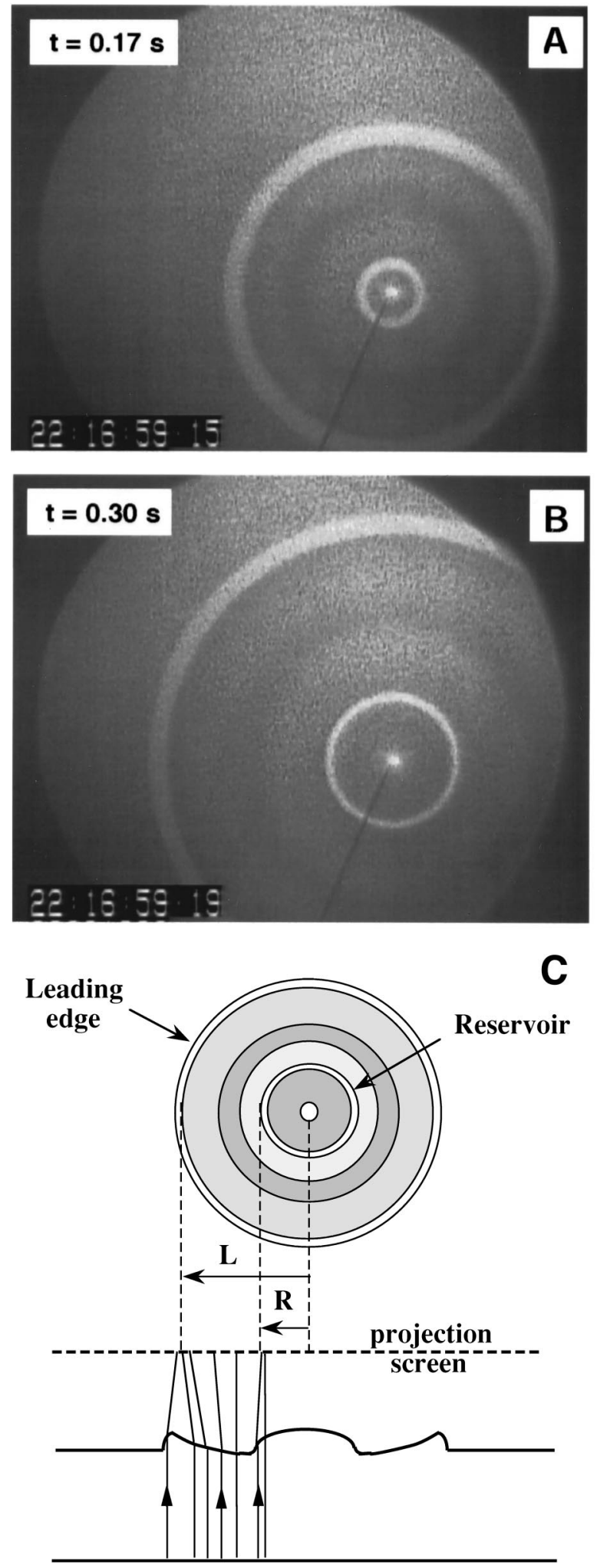

FIG. 3. Shadowgraph images produced by optical assembly in Fig. 1. (a) and (b): silicone oil $(V \simeq 4 \mu l)$ spreading on water at two different times after deposition; (c): ray tracing and inferred surface profile.

transmitted light, their location could be observed simultaneously with the outermost white band seen in Fig. 4. The talc powder was pushed to the outer radius of this white band never penetrating into this elevated rim. This white band was 

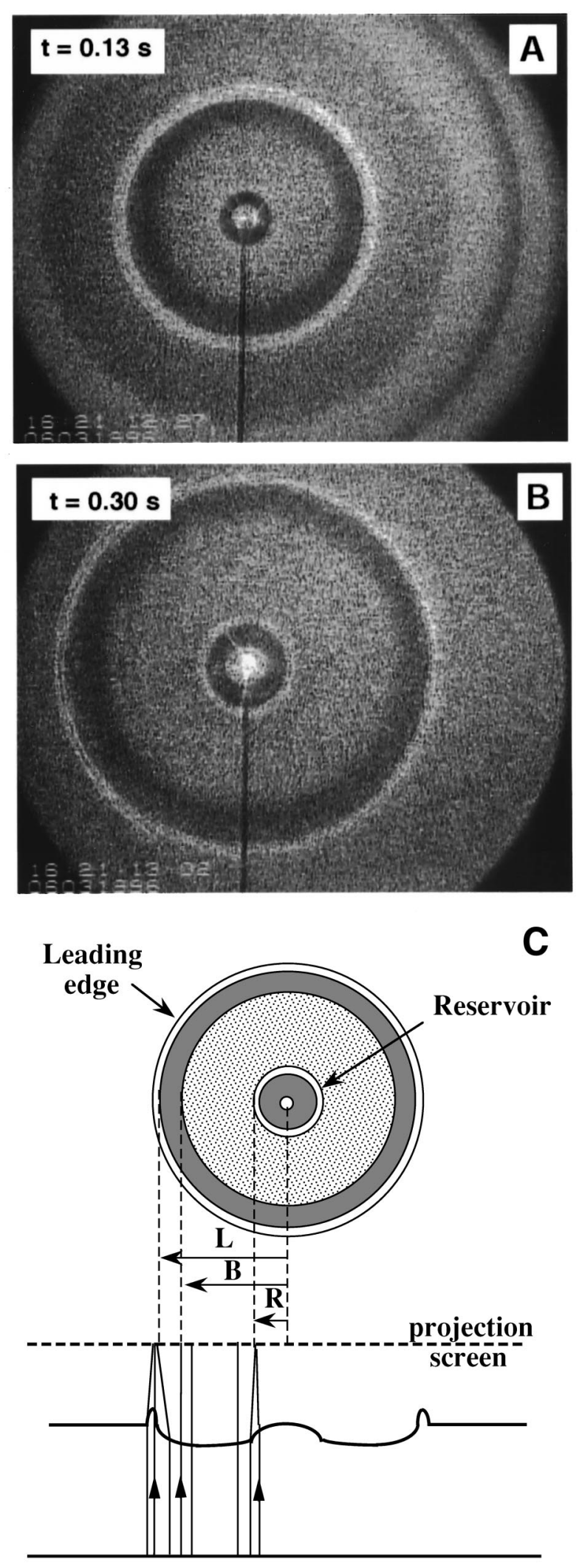

FIG. 4. Shadowgraph images produced by experimental assembly in Fig. 1. (a) and (b): toluene ( $V \simeq 3 \mu \mathrm{l})$ spreading on water at two different times after deposition; (c): ray tracing and inferred surface profile.

sometimes distorted from perfect circularity due to aggregating clumps of the talc powder, a practical concern which justifies the use of laser shadowgraphy alone in monitoring the location of moving liquid fronts.
Shadowgraphy is mainly a qualitative tool since the exact film thickness profile cannot be determined unambiguously. The light intensity is proportional to the second derivate of the film thickness so that different surface profiles can give rise to similar intensity patterns. It is nonetheless unambiguous that the largest white band in the shadowgraphs locates a thickened rim at the film front. Although the study of this elevated rim is beyond the scope of this paper and should be studied using optical techniques better suited to measurements of the surface shape, we have reviewed the literature to make contact with previous work reporting advancing rims in free surface flows. Many studies have investigated the shape of the so-called Thoreau-Reynolds ridge, ${ }^{21-25}$ whose existence was fully explained by Harper and Dixon in $1974 .^{26}$ As described by Scott and others, ${ }^{24}$ whenever surface active films accumulate near a surface barrier, they immobilize the surface and resist compression and dilation, forming a flexible surface film. A liquid stream of density, $\rho$, flowing towards the barrier at constant velocity, $U$, encounters the edge of this static and almost incompressible film of surface tension, $\gamma$, and is suddenly retarded by the viscous boundary layer which has formed underneath it. Inviscid pressure variations of order $\rho U^{2}$ deflect the surface of the uncontaminated water a height, $h$, determined by the balance $\rho U^{2} \sim \rho g h \sim \gamma \partial^{2} h / \partial x^{2}$, where the last term represents the Laplace pressure caused by surface curvature in the streamwise direction. $^{26}$ It has been argued in the literature $^{22,23}$ that this ridge should also form in front of a surface active film rapidly advancing over a quiescent water surface since an observer moving with the leading edge observes the identical flow conditions just described for the stationary monolayer. Studies in which the Reynolds ridge has been carefully measured, ${ }^{24,25}$ however, have used oncoming streams of constant velocity whereas the nonvolatile spreading films we have studied experience a constantly diminishing speed, $d L / d t \sim t^{-1 / 4}$, due to the diminished Marangoni stresses created during spreading. Though we cannot locate exactly the actual leading edge of the spreading film, the elevated front we observe could signal the presence of the Thoreau-Reynolds ridge. The elevation appears to occur at least partly, if not wholly, in the oil covered portion of the film. The elevation increases in the presence of evaporation suggesting once again that the elevation develops in the hydrocarbon covered portion of the water. Jensen ${ }^{9}$ has recently proposed a different mechanism by which the leading edge of a nonvolatile film can undergo significant deflections, with a strong depression behind the moving front and a surface elevation at the leading edge. Though these calculations do not include the effects of capillarity or gravity, they clearly demonstrate the possibility of surface elevation from Marangoni stresses and provide an explanation for surface deflection quite distinct from the formation of the ThoreauReynolds ridge. We intend to address this issue in future experiments using optical techniques more sensitive to the surface slope of the advancing film. In particular, careful measurements of the surface elevation as a function of time can distinguish between these two mechanisms. 
TABLE II. Power law fits to leading edge, $L(t)$, and reservoir edge, $R(t)$, for three independent experiments of silicone oil spreading on water.

\begin{tabular}{|c|c|c|c|c|c|c|c|c|}
\hline \multirow[t]{2}{*}{$\begin{array}{c}\text { Expt. } \\
\text { no. }\end{array}$} & \multicolumn{2}{|c|}{$\begin{array}{c}L=k_{L} t^{\alpha_{L}} \\
\text { (A) }\end{array}$} & \multirow{2}{*}{$\begin{array}{c}L=k_{L}^{*} t^{3 / 4} \\
(\mathrm{~B}) \\
k_{L}^{*}\end{array}$} & \multicolumn{2}{|c|}{$\begin{array}{c}R=k_{R} t^{\alpha_{R}} \\
\quad \text { (C) }\end{array}$} & \multicolumn{3}{|c|}{$\begin{array}{c}\text { Linear } \\
\text { correlation } \\
\text { coefficient }\end{array}$} \\
\hline & $k_{L}$ & $\alpha_{L}$ & & $k_{R}$ & $\alpha_{R}$ & (A) & (B) & (C) \\
\hline I & 8.58 & 0.708 & 9.00 & 3.17 & 0.921 & 0.998 & 0.997 & 0.994 \\
\hline II & 8.76 & 0.844 & 7.96 & 3.08 & 0.950 & 0.999 & 0.993 & 0.996 \\
\hline III & 8.08 & 0.671 & 8.26 & 3.36 & 1.053 & 0.999 & 0.995 & 0.999 \\
\hline Average & 8.48 & 0.741 & 8.40 & 3.20 & 0.975 & $\ldots$ & $\ldots$ & $\ldots$ \\
\hline
\end{tabular}

\section{B. Control study with nonvolatile films: spreading of silicone oil}

To our knowledge laser shadowgraphy has not been used to visualize the spreading of thin films on a deep liquid layer. We therefore used our apparatus first to measure the leading edge of pure silicone oil spreading on water. Silicone oil is an excellent control sample since it neither evaporates nor dissolves in water. For these reasons, it has been used in many spreading experiments to confirm the scaling law for nonvolatile systems given by Eq. (3)., ${ }^{3,713,27,28}$ All previous experiments were conducted in rectilinear geometry with a constant concentration source provided by an infinite reservoir. Our spreading experiments also use an effective infinite reservoir (on the time scale of our measurements) but the spreading occurs in axisymmetric geometry. Equation (3) is equally valid for axisymmetric geometries but the coefficient $K$ is smaller than in rectilinear geometry since the spreading material must cover an ever widening area. The coefficient $K$ for silicone oil spreading on water in axisymmetric geometry is not available in the literature but similarly behaving oils have been tested in this geometry. ${ }^{13}$ We compare our results with these relevant studies.

Two typical shadowgraphs for a spreading silicone oil droplet are shown in Fig. 3 at times $t=0.17 \mathrm{~s}$ and $t=0.3 \mathrm{~s}$ after deposition. The radius, $L(t)$, was measured by a fitting routine which draws a circle through three randomly selected points on the periphery of the ring. The rapid spreading speed causes the leading edge to fall out of view within $1 / 2$ s. The data obtained within this short time period, however, allows a good fit to power law behavior. We fitted the data collected from three spreading experiments in two different ways with slightly different results as shown in Table II. The column labeled (A) represents a least-squares fit of the data to the form $L=k_{L} t^{\alpha_{L}}$ where both $k_{L}$ and $\alpha_{L}$ are adjustable parameters. The column labeled (B) represents a leastsquared fit to the form $L(t)=k^{*}{ }_{L} t^{3 / 4}$ where the only adjustable parameter is $k^{*}{ }_{L}$. The bottom row lists the average values for the three spreading experiments. As shown in column (A), the full two parameter fit yields an average value of 0.741 for the spreading exponent of silicone oil advancing over water. This value is in excellent agreement with the theoretical prediction of $3 / 4$.

Comparison of the overall coefficient between the two fitting forms yields similar values, namely $k_{L}=8.48$ for the two parameter fit and $k^{*}{ }_{L}=8.40$ for the single parameter fit. According to Eq. (3) the overall prefactor in the spreading law depends on the spreading coefficient and the density and viscosity of the support liquid according to $k_{L}$ $=K S^{1 / 2} /(\mu \rho)^{1 / 4}$. By substituting the values for silicone spreading on water we find $K=0.88$ (for the two parameter fit) and $K=0.87$ (for the single parameter fit). $\mathrm{Camp}^{13}$ has compiled all available theoretical and experimental values for $K$. Theoretical predictions for the value of $K$ in unidirectional geometry have varied between 0.665 and 1.52 depending in part on assumptions regarding the constitutive behavior of the film. Experimental values obtained from the spreading of single or multicomponent oils and alcohols have ranged between $1.1 \leqslant K \leqslant 2.1$. Camp's own careful experiments of different spreading oils in unidirectional geometry produced values in the range $1.27 \leqslant K \leqslant 1.45$, while preliminary experiments in axisymmetric geometry produced coefficients in the range 0.67-1.06 (see Appendix A in Ref. 13). Since our calibration studies with silicone oil are in good agreement with Camp's preliminary measurements in axisymmetric geometry, we conclude that despite the short measurement interval allowed by our assembly $(t \lesssim 0.5 \mathrm{~s})$, the laser shadowgraphy technique accurately captures the behavior of the leading edge of a spreading film. We note, however, that even under stringent conditions of cleanliness, the value of $3 / 4$ for nonvolatile systems is not always reproducible from a single run due to the small spreading area and the limited time interval available with our test cells $(8 \mathrm{~cm}$ in radius). Three separate runs were required to produce good agreement with the $3 / 4$ spreading exponent.

Besides the white ring $L(t)$ there exists another white band at the droplet periphery labeled $R(t)$ whose temporal growth is also included in Table II. The location of this moving edge was nicely fit by a power law of the form $R(t)$ $=k_{R} t^{\alpha_{R}}$ with average values for the coefficient and exponent given by $k_{R}=3.20$ and $\alpha_{R}=0.975$. Since this moving boundary has not before been reported in the literature, more experiments are required to determine these values more exactly. For example, it would be interesting to study if this spreading exponent is closer to a value of one. Such additional studies could determine the dynamics of by which the macroscopic droplet delivers material to the thin spreading film downstream. From these limited measurements it appears nonetheless that the spreading behavior near the source region is different than the spreading dynamics downstream. 


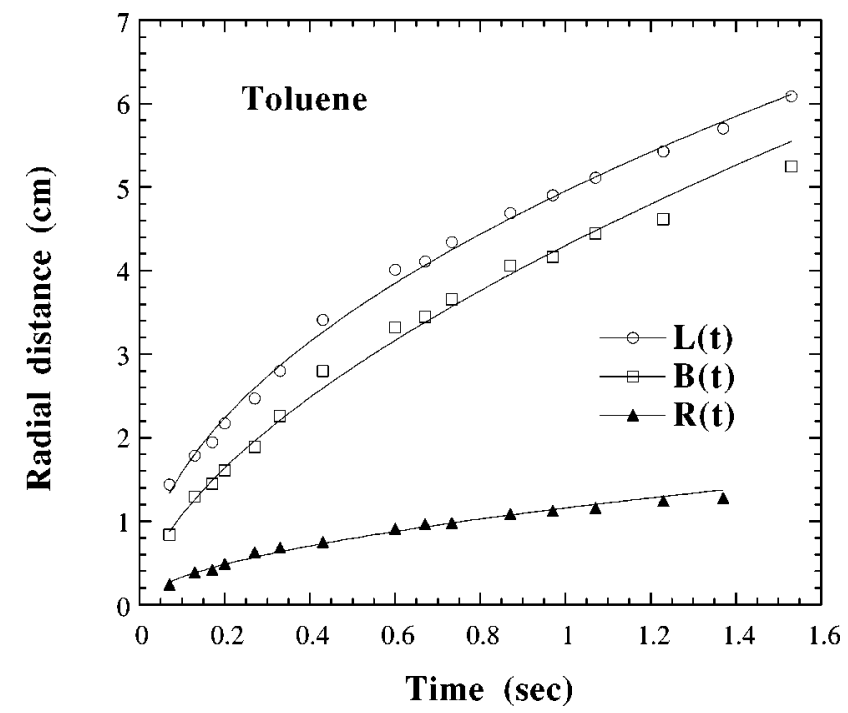

FIG. 5. Time evolution of bands $L(t), B(t)$, and $R(t)$ for toluene ( $V$ $\simeq 3 \mu$ l) spreading on water in an unsaturated atmosphere.

\section{Spreading of volatile films}

Figure 4 displays a shadowgraph of a toluene droplet spreading on a deep water support. Due to the higher curvature of the moving fronts in the volatile systems, the three distinct fronts, $L(t), B(t)$, and $R(t)$ could easily be measured over a time interval of approximately $2 \mathrm{~s}$. As compared to the silicone oil studies, the slower spreading speeds of the volatile films allowed data to be collected over a longer time period. Unlike the silicone oil, however, we noted that after a time interval of a few seconds, the radius of the reservoir droplet for toluene and xylene, labeled $R(t)$ in Fig. 4(c), stopped advancing and began retracting from the water surface in a rapid and spontaneous manner. This receding edge underwent a peculiar fingering instability with a distinct wavelength followed by a period of turbulent mixing and retraction. ${ }^{29}$ During this period of reservoir dewetting, the leading edge $L(t)$ continued to advance smoothly and steadily over the water surface until reaching the cell walls. Despite this smooth advance, we present here only the re- sults from the initial stage of spreading (during the period $t$ $\lesssim 2$ s for the minute volumes used) during which the reservoir was well behaved and stable.

The evolution rate of the three bands was measured as before by fitting a circle to three random points along the periphery of each ring. As an example, the temporal advance of all three bands for a toluene droplet is shown in Fig. 5. Toluene has the largest spreading coefficient of the liquids tested and spreads the most rapidly. In Table III we list the results of all the volatile spreading experiments, each event lasting no more than $2 \mathrm{~s}$. Because of the very low contrast exhibited by $n$-heptane in the region near $B(t)$, this boundary could not be measured with good accuracy and is omitted from the list. Surprisingly, each moving boundary displays power law behavior. As shown in Table III, all the data was successfully fit to the functional form $r_{i}=k_{i} t^{\alpha_{i}}$ (where $i$ $=L, B$, or $R$ ) for the three different spreading radii $r_{i}(t)$. The parameters $k_{i}$ and $\alpha_{i}$ represent the two adjustable parameters whose values are determined form a least-squared fit. The standard deviation for the values of $k_{L}$ and $k_{B}$ is typically $3 \%$ to $4 \%$, while for $k_{R}$ the value is closer to $8 \%$. This reproducibility is quite good given the fact that we are deriving exponents over a limited range in time and that surface studies with a high surface tension liquid like water can easily suffer extraneous contamination.

The spreading behavior of the leading edge for all volatile liquids tested is shown in Fig. 6 along with the results from the silicone oil experiments. The solid lines represent the average power law behavior from the least-squares fit presented in Table III while individual icons for each substance represent data collected from three arbitrary runs. Remarkably, even in the presence of evaporation the leading edge advances as a power law in time for all the volatile films examined. Within experimental error, this exponent assumes a value of $1 / 2$, significantly lower than the $3 / 4$ exponent produced by the silicone oil films. This behavior may signal a universal scaling law for deep layer Marangoni driven spreading coupled to film evaporation.

The time evolution of the edge of the droplet reservoir, $R(t)$, is plotted in Fig. 7. The solid lines represent the average exponent in Table III while individual icons for each

TABLE III. Power law fits to bands $L(t), B(t)$, and $R(t)$ (measured in $\mathrm{cm}$ ) for four volatile hydrocarbon films spreading on water in an unsaturated atmosphere. $N=$ number of independent spreading experiments, $\alpha_{i}=$ spreading exponent of band $i, k_{i}=$ overall spreading prefactor, and $r=$ linear correlation coefficient for the two parameter fit.

\begin{tabular}{|c|c|c|c|c|c|c|c|c|c|c|c|c|}
\hline \multirow[t]{2}{*}{ Liquid } & \multicolumn{4}{|c|}{ Leading edge $L(t)$} & \multicolumn{4}{|c|}{ Boundary $B(t)$} & \multicolumn{4}{|c|}{ Reservoir $R(t)$} \\
\hline & $N$ & $\alpha_{L}$ & $k_{L}$ & $r$ & $N$ & $\alpha_{B}$ & $k_{B}$ & $r$ & $N$ & $\alpha_{R}$ & $k_{R}$ & $r$ \\
\hline toluene & 10 & $\begin{array}{c}0.49 \pm \\
0.05\end{array}$ & $\begin{array}{c}4.86 \pm \\
0.27\end{array}$ & 0.999 & 20 & $\begin{array}{c}0.60 \pm \\
0.04\end{array}$ & $\begin{array}{c}4.38 \pm \\
0.21\end{array}$ & 0.997 & 20 & $\begin{array}{c}0.53 \pm \\
0.05\end{array}$ & $\begin{array}{c}1.14 \pm \\
0.10\end{array}$ & 0.994 \\
\hline$p$-xylene & 10 & $\begin{array}{c}0.47 \pm \\
0.02\end{array}$ & $\begin{array}{c}5.15 \pm \\
0.16\end{array}$ & 0.999 & 20 & $\begin{array}{c}0.58 \pm \\
0.05\end{array}$ & $\begin{array}{c}4.63 \pm \\
0.23\end{array}$ & 0.996 & 20 & $\begin{array}{c}0.52 \pm \\
0.05\end{array}$ & $\begin{array}{c}1.63 \pm \\
0.13\end{array}$ & 0.990 \\
\hline $\begin{array}{c}2,2,4 \\
\text { trimethyl- } \\
\text { pentane }\end{array}$ & 10 & $\begin{array}{c}0.47 \pm \\
0.03\end{array}$ & $\begin{array}{c}3.83 \pm \\
0.15\end{array}$ & 0.997 & 11 & $\begin{array}{c}0.57 \pm \\
0.03\end{array}$ & $\begin{array}{c}3.29 \pm \\
0.13\end{array}$ & 0.996 & 11 & $\begin{array}{c}0.45 \pm \\
0.03\end{array}$ & $\begin{array}{c}1.14 \pm \\
0.11\end{array}$ & 0.991 \\
\hline$n$-heptane & 13 & $\begin{array}{c}0.48 \pm \\
0.04\end{array}$ & $\begin{array}{c}3.22 \pm \\
0.11\end{array}$ & 0.993 & $\cdots$ & $\cdots$ & $\cdots$ & $\cdots$ & 13 & $\begin{array}{c}0.36 \pm \\
0.02\end{array}$ & $\begin{array}{c}1.04 \pm \\
0.07\end{array}$ & 0.996 \\
\hline
\end{tabular}




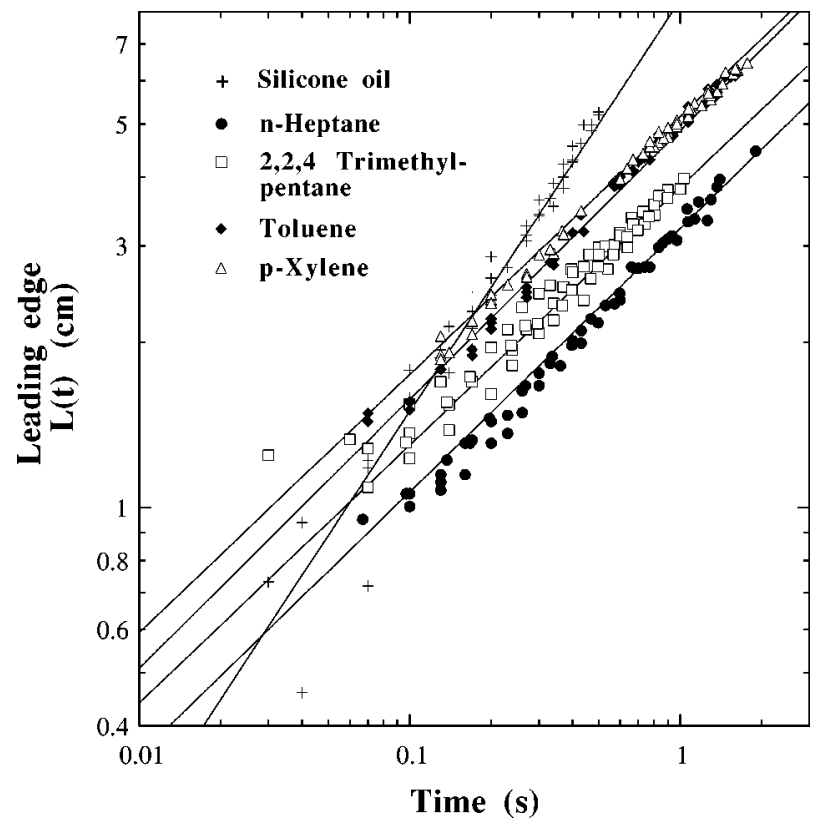

FIG. 6. Radial advance of leading edge, $L(t)$, for volatile liquids and nonvolatile silicone oil spreading over water surface in an unsaturated atmosphere. Solid lines represent a power law fit to the data, $L=k_{L} t^{\alpha_{L}}$, where values of $k_{L}$ and $\alpha_{L}$ are listed in Tables II and III.

substance represent data collected from three arbitrary runs. A comparison of the data in Table III reveals that the reservoir typically advances three to four times slower than the leading edge. The spreading exponent $\alpha_{R}$ ranges in value from 0.35 for $n$-heptane to approximately 0.5 for toluene, $p$-xylene, and trimethylpentane. The exponents $\alpha_{L}$ and $\alpha_{R}$ are very similar in value for toluene, $p$-xylene, and trimethylpentane, but the value of $\alpha_{R}$ for $n$-heptane is much smaller than $\alpha_{L}$. In addition, $n$-heptane produces the smallest coefficient $k_{R}$. According to Table I, $n$-heptane is highly volatile and displays a very small spreading coefficient on water. Perhaps evaporation/condensation at the droplet location is

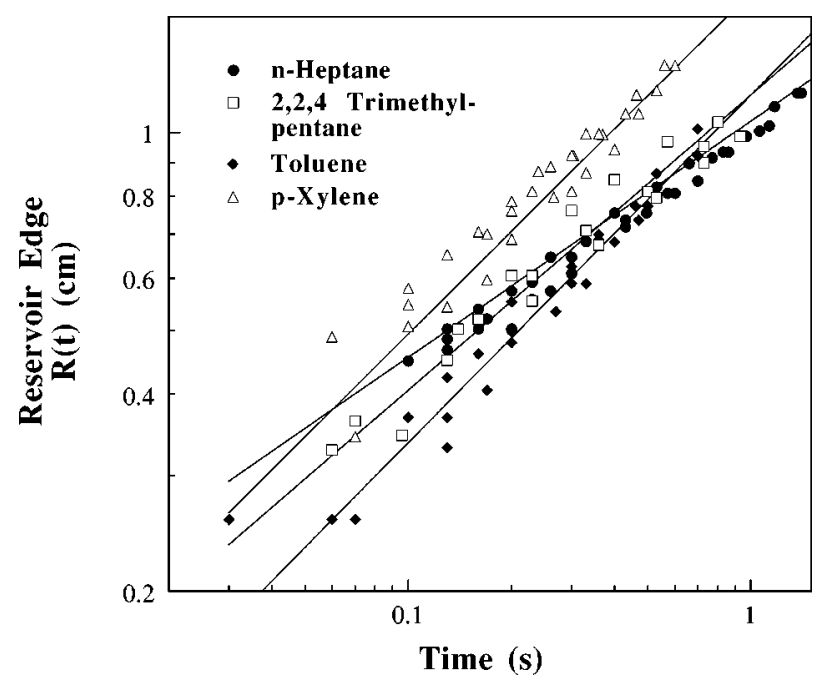

FIG. 7. Measurement of $R(t)$ for droplets $(V \simeq 3-5 \mu 1)$ of volatile liquids spreading on water in an unsaturated atmosphere. Solid lines represent the average power law fit to the data, $R=k_{R} t^{\alpha_{R}}$, where values of $k_{R}$ and $\alpha_{R}$ are listed in Table III.
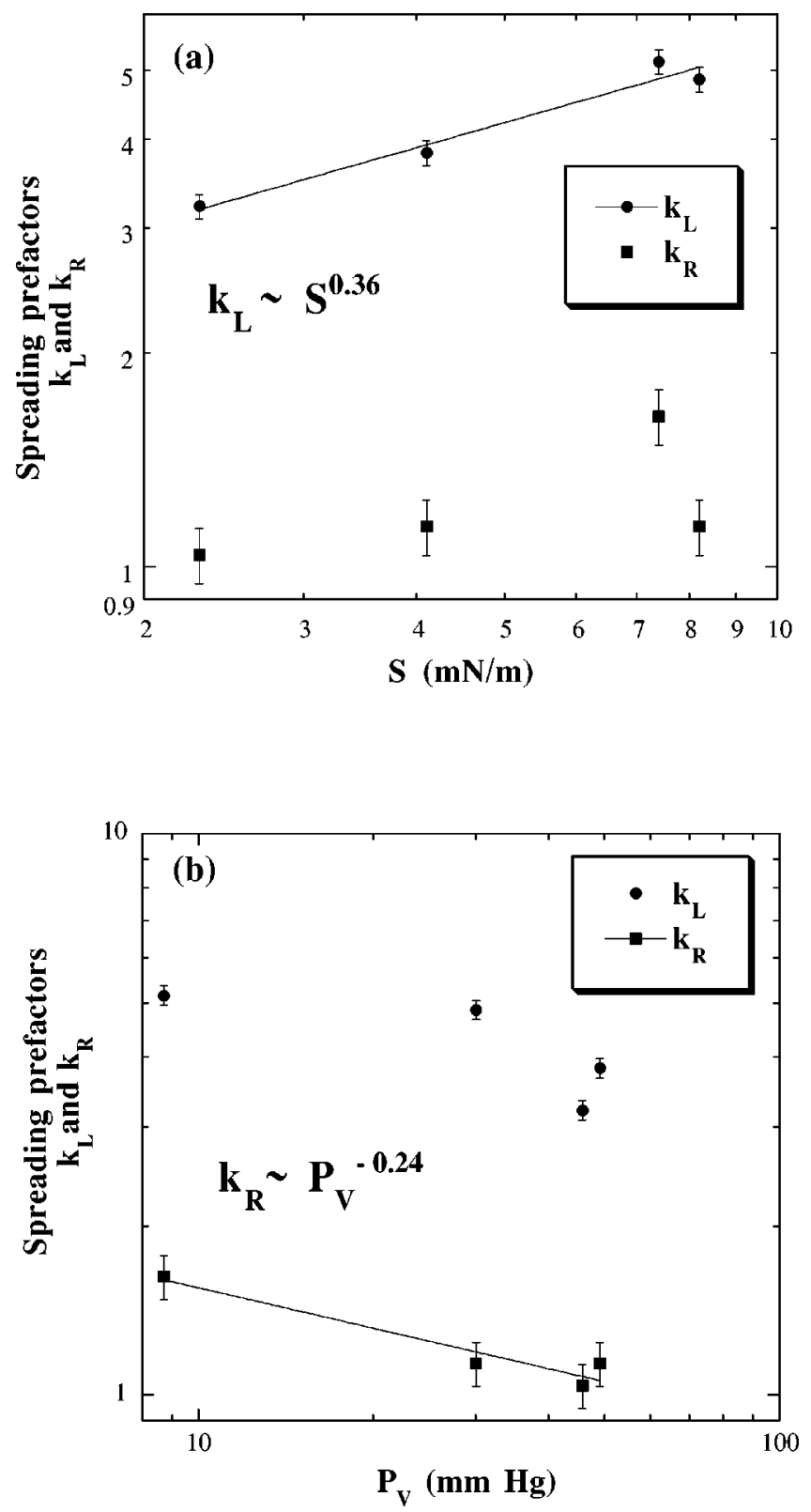

FIG. 8. Correlation of $k_{L}$ and $k_{R}$ with (a) spreading coefficient, $S$, and (b) vapor pressure, $P_{V}$, for different volatile liquids.

affecting the advance of $R(t)$ and slowing both its spreading coefficient and exponent. In line with this trend, we also noted that $k_{R}$ is largest for xylene, the hydrocarbon with the lowest vapor pressure of the volatile liquids tested.

With the addition of film evaporation, the spreading prefactors $k_{i}$ cannot simply be deconvoluted in a manner analogous to Eq. (3). Since there is no theoretical model in the literature for the influence of $S$ and $P_{V}$, the film vapor pressure, on the spreading dynamics of a thin film, we attempted to find some empirical trends between $k_{L}$ and $k_{R}$ and these two material parameters. In Figs. 8(a) and 8(b) we plot the correlation of $k_{L}$ and $k_{R}$ against $S$ and $P_{V}$. The coefficient $k_{R}$ does not correlate well with $S$; however, $k_{L}$ increases with increasing $S$, as expected if the driving force for the leading edge derives from the spreading coefficient, $S$. We have fit this rise to the relation $k_{L} \sim S^{0.36}$, though more 

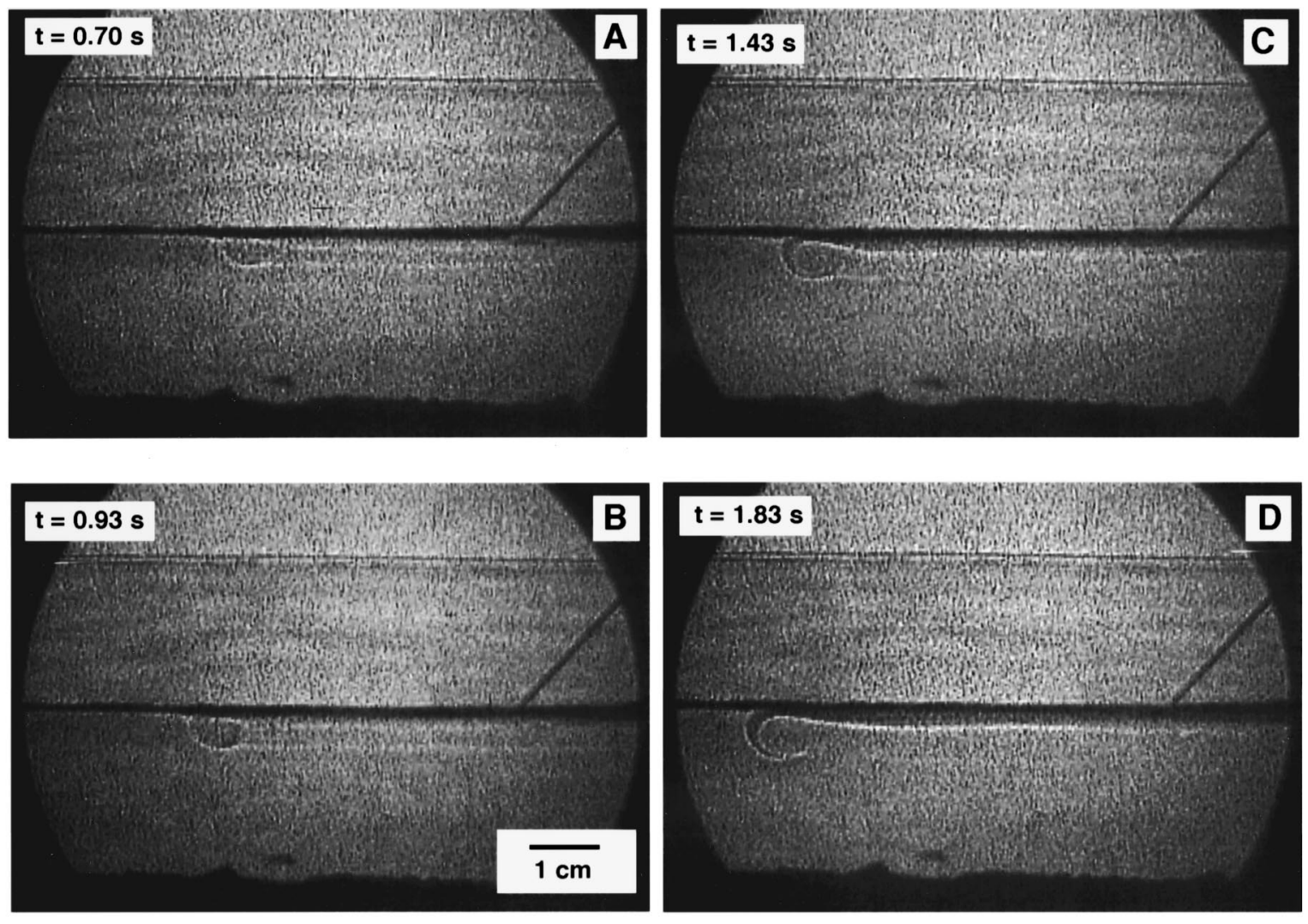

FIG. 9. Sideview shadowgraphs $(0.7 \mathrm{~s} \lesssim t \lesssim 1.83 \mathrm{~s})$ for toluene spreading on water produced by directing a horizontal collimated beam of light near the air-liquid interface.

volatile liquids, whose spreading coefficients on water span more than a decade, need to be examined in order to secure an accurate value for this exponent. In comparing the rate of advance of nonvolatile with volatile liquids, the former advance as $S^{1 / 2}$ from Eq. (3), somewhat stronger than the correlation for volatile substances. In Fig. 8(b) we have plotted the correlation of $k_{L}$ and $k_{R}$ with the vapor pressure $P_{V}$. There is no evident correlation between $k_{L}$ and $P_{V}$, but a clear correlation of $k_{R}$ with $P_{V}$ which we have fit to the form $k_{R} \sim P_{V}{ }^{-0.24}$. Once again, more samples must be tested to secure the exact value of this exponent. Further studies are being conducted to elucidate the trends in the data which suggest that the rate of advance of the leading edge is controlled by the spreading coefficient $S$ while the rate of advance of the reservoir is more closely linked to the vapor pressure.

\section{Visualization of subsurface flow}

\section{Shadowgraph imaging}

The well-known 3/4 exponent describing the location of the leading edge for nonvolatile, immiscible films spreading from an infinite reservoir derives from the presence of a laminar viscous boundary layer established by the rapidly spreading surface film. Since the spreading exponent in volatile systems is much reduced, we investigated the flow behavior beneath the volatile spreading films to gain an understanding of differences induced by the presence of evaporation. To visualize the flow pattern which develops in the water subphase, we employed both sideview laser shadowgraphy and seeding of the bulk water support with highly reflective aluminum flecks. The sideview shadowgraphy detects strong variations in the refractive index caused by density variations in the bulk fluid induced by compositional or thermal effects. An estimate of the thickness of the spreading film from the volume of hydrocarbon deposited and the areal coverage indicates that the volatile film is typically less than a micron in thickness. Since the density variations observed occur on the scale of millimeters, the intensity variations observed on the projection screen must develop not in response to concentration variations but in response to significant vertical temperature gradients in the water sublayer.

The four time-lapse photographs shown in Fig. 9 clearly display the subsurface motion for toluene spreading on water. The syringe needle used for deposition appears to the right in each photograph. The top rim of the glass cell which houses the water support appears at the top of each frame. The rough bottom surface of the cell is created by the silicone sealant used to assemble the five sides of the rectangular tank. The thick black region beneath the spreading film denotes a region undergoing sharp changes in the refractive index, $n$, where the spatial variations, $\partial^{2} n / \partial z^{2}$, strongly deflect the transmitted light away from its original trajectory. While the subsurface motion during the spreading of silicone oil is featureless, spreading volatile films develop an unusual thermal layer in the water support. In Fig. 9, approximately $0.7 \mathrm{~s}$ after the toluene droplet touches the water surface, two sharp bright lines trace out a wide horizontal strip of thick- 


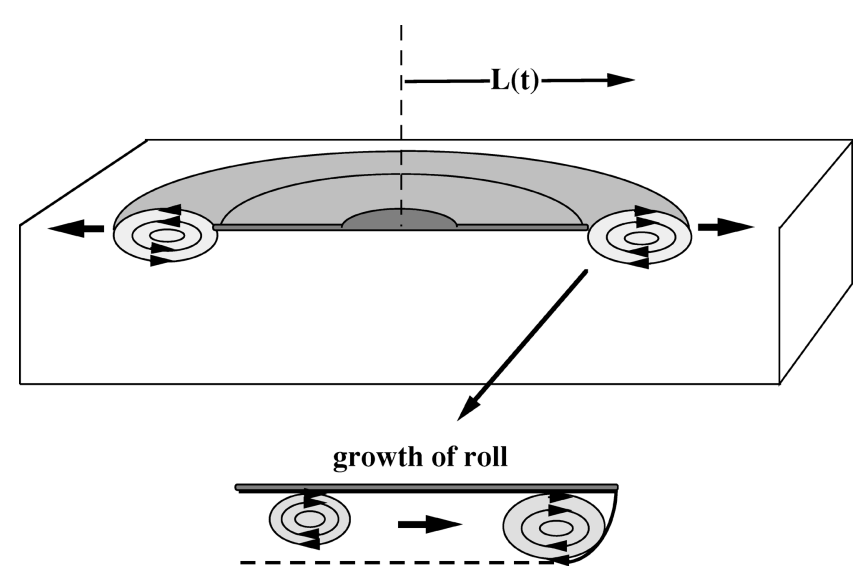

FIG. 10. Schematic of radially advancing convective roll beneath a spreading volatile film. Magnified view of stretched roll which produces flat thermal layer.

ness $t \approx 5 \mathrm{~mm}$ beneath the surface toluene film. This region is capped by an elliptically shaped roll beneath the leading edge.

To better visualize this flow profile, we conducted separate measurements in which the entire water support was seeded with highly reflective aluminum particles and illuminated by a vertical laser sheet. These experiments confirmed that the ellipsoidal fluid element represents the cross section of a localized rotational flow moving in horizontal fashion right beneath the free surface in tandem with the moving front. This circulatory flow is not a vortex but a radially advancing roll concentric with the origin where the droplet is initially deposited, as sketched in Fig. 10. The roll is ellipsoidal and not circular in cross section probably because it develops within an overall shear flow. As shown in the lower part of Fig. 10, the advancing roll traces out a flat horizontal thermal region. As discussed in greater length in the next section, the two bright lines and the development of the convective roll are believed to be caused by the evaporation and cooling of the rapidly spreading volatile film. This circulatory flow pattern requires some time to develop. In the toluene photographs shown in Fig. 9, the convective roll only appears onscreen after approximately $0.7 \mathrm{~s}$. This time lag may be attributed to two different reasons. There is always present a water meniscus on the walls of the cell which prevents reliable observation of the fluid within the first $3 \mathrm{~mm}$ or so of the air-liquid interface. Perhaps the thermal lines only become visible when the thickness of the cooled fluid layer has exceeded $3 \mathrm{~mm}$. A second possibility is that an observable thermal layer may not develop until the surface film has spread to a critical radius to produce sufficient surface cooling and a significant change in the water's refractive index. The thickness of the cooled layer increases with time and remains practically flat except at the leading edge, where the convective roll steadily increases in size. As shown in Figs. 9(c) and 9(d), it appears that as the first bright line begins fading, a second bright line appears above the first. We do not understand the development of this second line.

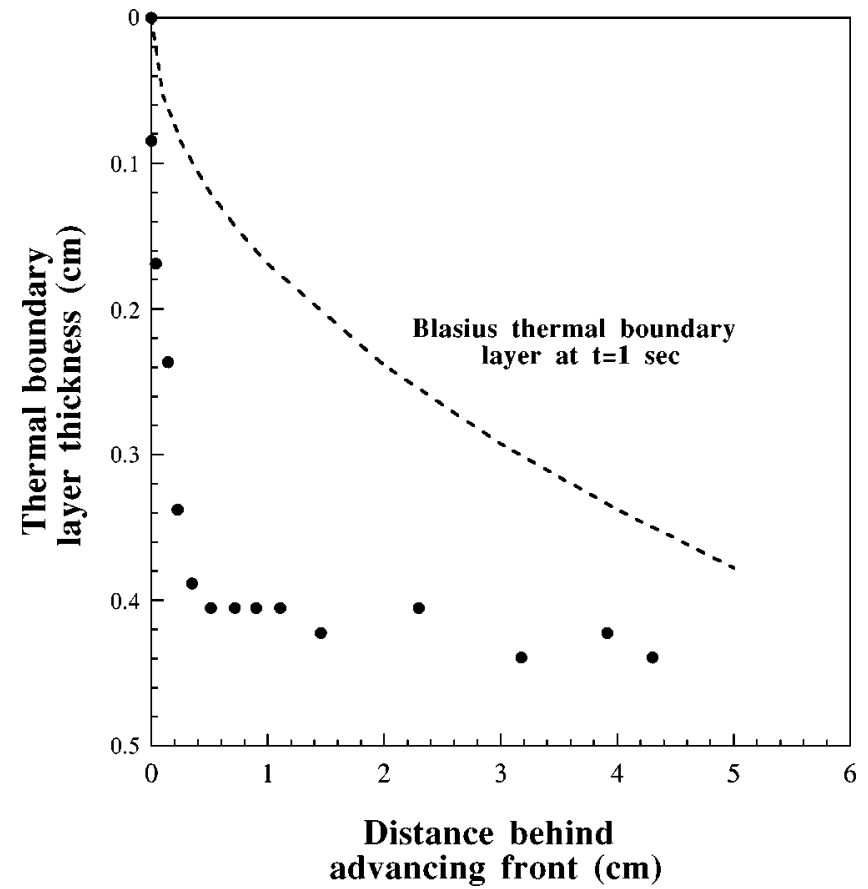

FIG. 11. Comparison of shape of thermal layer observed in Fig. 9 with Blasius thermal boundary layer for fixed instant in time.

\section{Geometric aspects of the subsurface thermal boundary layer}

Previous work by O'Brien et al. ${ }^{30}$ on the spreading of oleic acid on water has shown that the motion in the water subphase during the spreading of a thin nonvolatile immiscible film resembles a laminar viscous boundary layer. This study is one of the few in the literature to have visualized the shape and velocity profile of the bulk water dragged along by a rapidly spreading surface film. Unfortunately, comparisons between experiment and the velocity profiles expected from a Blasius viscous boundary layer theory were not made. For the most part, researchers have implicitly assumed the presence of a laminar viscous boundary layer because of the excellent agreement between experiments and the expression for the location of the leading edge given by Eq. (3).

In the absence of any instabilities and in the presence of simultaneous heat and momentum transfer in the water support, a laminar Blasius thermal boundary layer, $\delta_{\Delta T}$, should develop whose thickness is smaller than the viscous boundary layer, $\delta$, by a factor $\operatorname{Pr}^{1 / 3}$, where $\operatorname{Pr}$ represents the Prandtl number of the subphase liquid, according to

$$
\delta_{\Delta T}=\frac{5.0}{\operatorname{Pr}^{1 / 3}}\left(\frac{\nu x}{U}\right)^{1 / 2} .
$$

In this equation, $\nu$ represents the kinematic viscosity of the subphase liquid, $x$ the distance behind the advancing front, and $U=d L / d t$ the velocity of the advancing front. The viscous boundary layer is here defined in the frame of reference of the advancing film as the distance beneath the film where the longitudinal velocity reaches $99 \%$ of the free stream velocity $U$. We compared the shape and evolution of the thermal layer observed in Fig. 9 with the shape and evolution of a Blasius thermal boundary layer. The upper curve in Fig. 11 


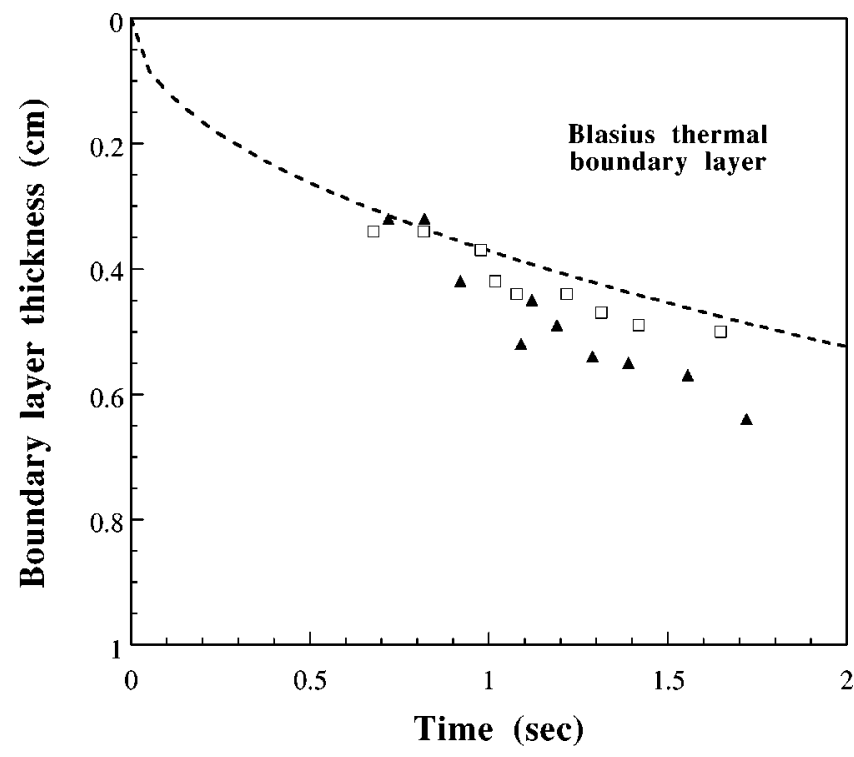

FIG. 12. Comparison of the temporal evolution of a flat portion of the thermal layer in Fig. 9 with the Blasius thermal layer at a fixed position $x$ $=L$ behind the leading edge.

represents a snapshot of the shape of a Blasius thermal layer which would develop in the water subphase after a period of $1 \mathrm{~s}$ if the spreading film could simply be regarded as a cool uniformly moving plate advancing over a warmer water surface. The thickness of this thermal boundary layer for water at $20{ }^{\circ} \mathrm{C}$ was estimated from the parameter values $\mathrm{Pr}$ $=6.96$ and $\nu=0.995 \times 10^{-2} \mathrm{~cm}^{2} / \mathrm{s}$. The values of $x$ and $U$ $=d L / d t$ were calculated from the actual position and speed of the toluene front from the fitted equation $L(t)=4.86 t^{0.49}$ in Table III to give the values at $t=1 \mathrm{~s}$ of $x=4.86 \mathrm{~cm}$ and $U=2.38 \mathrm{~cm} / \mathrm{s}$. The experimental data represents the thickness of the thermal layer measured directly from shadowgraph images. Since shadowgraphy is only sensitive to the second derivate in the refractive index, it cannot locate exactly the actual boundary of the thermal layer which may lie slightly inside or outside the contour shown. As evident in Fig. 11, however, the observed thermal layer assumes a much flatter profile away from the leading edge than a Blasius thermal boundary layer.

Besides comparing the shapes of these profiles at one instant in time, we also compared the temporal evolution of the thermal layer at the location $x=L(t)$ corresponding to the position defined by the point of deposition. In Fig. 12 the experimental points represent the growth of the flat portion of the thermal layer during a time interval $0.7 \mathrm{~s} \lesssim t \lesssim 1.6 \mathrm{~s}$ for two independent spreading events. The theoretical curve represents the temporal growth of the thickness of the Blasius thermal boundary layer beneath the point of deposition [i.e., $x=L(t)]$. These theoretical values were estimated by substituting $x=L(t)=4.86 t^{0.49}$ and $U=d L / d t \equiv L_{t}$ into Eq. (4) to give $\delta_{\Delta T}(t)=5.0\left(\nu t / \alpha_{L}\right)^{1 / 2} / \mathrm{Pr}^{1 / 3}$. Within experimental error, the thickness of the flat thermal layer appears to increase at the same rate as the thickness of a Blasius layer. This agreement is not surprising since $\delta_{\Delta T}(L)$ scales as $\nu L / L_{t}$, which for any front advancing with a power in time, $L$ $=k_{L} t^{\alpha_{L}}$, will always produce the scaling $\delta_{\Delta T} \sim t^{1 / 2}$. This cor-
TABLE IV. Dimensions of propagating sublayer convective roll. Horizontal extent $=l$ and vertical extent $=d$.

\begin{tabular}{cccc}
\hline \hline$L(\mathrm{~cm})$ & $l(\mathrm{~cm})$ & $d(\mathrm{~cm})$ & $l / d$ \\
\hline 5.2 & 0.76 & 0.42 & 1.8 \\
5.0 & 0.96 & 0.52 & 1.8 \\
5.3 & 0.78 & 0.52 & 1.5 \\
4.9 & 0.84 & 0.50 & 1.7 \\
\hline \hline
\end{tabular}

respondence simply reflects the role of thermal conduction and convection in determining the rate of heat transfer from the warmer bulk water to the cooler air-liquid interface.

Figure 11 clearly illustrates that the thermal boundary layer beneath the volatile spreading film is not of the Blasius type. Not only is the profile very flat behind the moving front but there exists a region of fluid circulation at the leading edge. We have measured the shape and extent of this stretched roll as it advances beneath the water surface. Since these measurements are taken directly from shadowgraphs, which cannot locate precisely the boundaries of the convection pattern, they serve mostly as estimates of the region of the flow affected by thermal gradients. In Table IV we list results from four different experiments for toluene spreading on water. The aspect ratio of the roll was measured $1 \mathrm{~s}$ after drop deposition since the roll did not appear until $0.7 \mathrm{~s}$ had elapsed. The horizontal extent of the roll is denoted by $l$ and its vertical extent by $d$. The horizontal extent was more difficult to measure due to the low contrast on the side farthest from the leading edge. This ambiguity may have led to the larger variations in this measurement. The average aspect ratio, $l / d$, for the development of the roll after $1 \mathrm{~s}$ of spreading was measured to be 1.7 , indicating significant "stretching', in the streamwise direction. Although these studies represent the flow behavior for toluene on water, we have observed similar phenomena at the leading edge of $p$-xylene whose vapor pressure is smaller than toluene. We suspect that many spontaneously spreading volatile films will exhibit the development of this convective roll whenever the surface cooling effect is significant. We are presently investigating in more detail the formation of the convective roll $^{31}$ using a novel laser-induced fluorescence flow tagging technique ${ }^{32}$ recently developed to visualize flow fields in water.

\section{Discussion and comparison with other cellular convective phenomena}

We reviewed the literature to find only one model in which researchers have coupled Marangoni driven spreading to evaporation. Camp ${ }^{13}$ treated the Marangoni driven spreading of volatile films along a deep fluid layer as a classical convection-diffusion process. The Marangoni stress establishes a viscous boundary layer beneath the spreading film while the evaporation process establishes a concentration boundary layer above the film. The spreading film is modeled as a moving rigid plate whose speed is determined from Eq. (3). The surface concentration of the moving plate is assumed constant in space and time. ${ }^{33}$ Both the momentum and concentration boundary layers are assumed to be quasisteady. The analysis predicts that the largest mass loss occurs 
at the advancing front at $x=0$ according to $J \sim P_{V}\left(U_{L} / x\right)^{1 / 2}$ where $J$ represents the mass loss in $\mathrm{mol} /\left(\mathrm{cm}^{2}-\mathrm{s}\right), P_{V}$ is the film vapor pressure which vanishes infinitely far from the film, $U_{L}$ is the velocity of the spreading film of length $L$, and $x$ the distance behind the leading edge. ${ }^{34}$ Assuming that the only effect of evaporation is to introduce mass loss into the spreading dynamics, then the spreading exponent of $3 / 4$ is unaffected. This analysis which predicts the amount of material lost to evaporation could be extended to link the mass loss to an increase in the spreading coefficient $S$ but this aspect has not been pursued in the literature. We expect that simple evaporative cooling of the surface with no distortion of the flow field in the water phase would produce a smaller value of $S$ thereby decreasing the spreading speed, but this effect would not necessarily change the spreading exponent unless there were some unusual time dependence introduced into $S$. For the solvents we used, $d \gamma / d T$ is approximately 0.2 $\mathrm{dyn} /{ }^{\circ} \mathrm{C}$. Even for temperature drops at the surface of the film as large as $5^{\circ} \mathrm{C}$, which is far in excess of the temperature drops produced during the spreading process, $S$ would only decrease by about $1 \mathrm{dyn} / \mathrm{cm}$. It appears unlikely that such a small decrease in the value of $S$ can produce the large measured difference in spreading exponents between nonvolatile and volatile films. In his experimental studies, Camp performed some preliminary spreading studies with slightly volatile fluids of very high spreading coefficients like nonanoic acid $\left(S=36 \pm 2\right.$ and $\left.P_{V}=1.0 \times 10^{-3} \mathrm{~mm} \mathrm{Hg}\right)$ and undecanol $\left(S=41.4 \pm 2\right.$ and $\left.P_{V}=6.8 \times 10^{-3} \mathrm{~mm} \mathrm{Hg}\right)$ and reported the usual $3 / 4$ spreading coefficient. Our studies with films of much lower spreading coefficients and high vapor pressures produce instead a spreading exponent closer to $1 / 2$ with a thermal boundary layer in the water subphase very different in shape than a Blasius profile. We conclude from our studies that although evaporative surface cooling can decrease the value of the spreading by a small amount, the presence of the fluid circulation at the leading edge introduces into the problem another significant source of viscous dissipation. The circulation in the sublayer obviates the possibility of a simple analysis based on laminar boundary layer flow since the rotational flow violates the assumptions inherent in the boundary layer approximation. The additional dissipation is expected to play a major role in the spreading dynamics and will surely decrease the speed of the advancing film. It is this aspect of the sublayer flow on which we focus in this work.

We hypothesize that the smaller exponent associated with the spreading of volatile liquids is linked to the additional viscous dissipation that occurs in the water sublayer due to the presence of the rotational convective flow. From the shadowgraphs it is evident that the coupling of Marangoni driven spreading and evaporation produces a significant temperature gradient in the water sublayer beneath the volatile spreading film. This temperature gradient can establish a convective instability similar to the development of a Rayleigh-Bénard instability in liquids heated from below or in evaporating and drying paint films in which the top surface of the film is effectively cooler than the interior. ${ }^{35}$ Our experiments with aluminum flecks indicate significant rotational flow beneath the leading edge with an upward flow behind the advancing rim where the surface is somewhat depressed and a downward flow at the front of the advancing rim. As first explained by Sternling and Scriven ${ }^{36}$ for steady cellular convection, purely Bénard instabilities caused by thermocapillary flow often display an upward flow where the surface is depressed and a downward flow where the surface is elevated in contrast to purely Rayleigh instabilities caused by buoyancy effects in which the upward flow occurs where the surface is most elevated and downward flow where the surface is depressed. Our particle seeding experiments trace out a flow pattern more like Bénard cellular convection but further experiments are being conducted to elucidate this issue. $^{31}$

We compare the aspect ratio of the propagating roll observed in our studies with similar measurements that have previously been made in true Rayleigh-Bénard experiments in which a initially quiescent fluid film bounded below by a heated plate is open to the atmosphere. Koschmieder's ${ }^{37}$ studies on the aspect ratio, $l / d$, of convective rolls (including both buoyancy and surface tension effects) in a finite container with a rigid conducting lower boundary produced values in the range 1.35-1.56, depending on the magnitude of the Biot number characterizing the heat transfer from the open upper surface. Our values shown in Table IV, ranging from $l / d=1.5-1.8$, are slightly larger indicating stretching in the horizontal direction. We attribute this stretching to the fact that the convective roll beneath the leading edge is superposed onto an overall shear flow caused by the Marangoni driven spreading process.

Unlike the conventional setup used to study RayleighBénard phenomena in which there develops many horizontal rolls, the volatile spreading films display only one roll during the time interval of measurement. The simplified model proposed by $\mathrm{Camp}^{13}$ for simultaneous heat and mass transfer can be used to understand why there appears only a single convective roll beneath the spreading film and why this roll appears beneath the leading edge and not further upstream. Within the approximations described earlier, the leading edge experiences the largest mass loss and therefore the largest cooling. In addition the thickness of the boundary layer is smallest at the leading edge. These two effects should produce the largest vertical temperature gradient in the water layer at that location. In order for a Rayleigh-Bénard convection cell to form, the system must exceed a critical Rayleigh or Marangoni number to produce cellular convection, both of which are directly proportional to the vertical temperature gradient. By this argument, if an instability should occur, it would first appear beneath the leading edge. Since the vertical temperature gradient decreases away from the leading edge, the brightness of the lines observed should decrease further back from the advancing front. The brightness of the thermal lines should also decrease in time since the slower the spreading velocity $d L / d t$, the smaller the overall evaporation rate and the less the surface cooling. Both of these features have been observed in our shadowgraphs.

Recently there has been reported in the literature one other example of a convective roll observed during the spreading of a nitroethane droplet in a saturated atmosphere 
on a deep water layer. Nitroethane is highly miscible with water. Using schlieren visualization in a study devoted to late stage interfacial turbulence of surface films, Santiago ${ }^{17}$ noted the presence of two advancing subsurface rolls during the spreading and dissolution process. One roll developed beneath the leading edge of the spreading film while a second developed beneath the droplet reservoir. Given the close analogy in the formation of Bénard cellular instabilities using either thermal or solutal driving forces, these recent experiments may indicate the possibility of convective rolls in either volatile or miscible spreading systems. Further studies are required to explore this intriguing analogy.

The most important difference between the boundary conditions in our studies and those traditionally used to study Rayleigh-Bénard convection is the presence of an overall shear flow on the convection motion as well as an additional space- and time-dependent horizontal temperature gradient at the surface of the volatile film due to the simultaneous spreading and evaporation. In the absence of any theoretical model for these different conditions, we have estimated the critical Rayleigh and Marangoni numbers calculated for different boundary conditions and in the absence of a shear field to estimate an order of magnitude for the vertical temperature gradient required to initiate a convective roll. For thermocapillary cells, the Marangoni number is defined to be

$$
M a=\frac{\partial \gamma}{\partial T} \frac{\Delta T d}{\rho \nu \kappa},
$$

where $\partial \gamma / \partial T$ represents the change in surface tension with temperature $T, \Delta T$ the temperature difference between the warmer lower surface and the cooler upper surface, $d$ the thickness of the fluid layer participating in the cellular convection, $\nu$ the kinematic viscosity, $\rho$ the fluid density, and $\kappa$ the thermal diffusivity of the heated fluid. In our estimate we use the critical value, $M a_{c}=80$, derived for a lower rigid surface and a free upper surface. ${ }^{35}$ Substituting a typical thermal boundary thickness from our shadowgraphs, $d=0.3 \mathrm{~cm}$, and the material constants for water at $20{ }^{\circ} \mathrm{C}$, namely $\nu=0.995 \times 10^{-2} \mathrm{~cm}^{2} / \mathrm{s}, \quad \kappa=0.143 \times 10^{-2} \mathrm{~cm}^{2} / \mathrm{s}, \quad \rho=0.998$ $\mathrm{g} / \mathrm{cm}^{3}$, and $\partial \gamma / \partial T=0.13 \times 10^{-3} \mathrm{dyn} / \mathrm{cm} \mathrm{K}$, the critical temperature difference for unstable flow is $\Delta T_{c}=0.03{ }^{\circ} \mathrm{C}$. Such a small temperature difference is easily achievable in evaporating systems. Since buoyancy effects may also help initiate the convective roll, we estimated the vertical temperature drop that would be required to induce a buoyancy driven instability as determined by the Rayleigh number:

$$
R a=\frac{\alpha g \Delta T d^{3}}{\nu \kappa},
$$

where $\alpha$ is the volume expansion coefficient and $g$ the gravitational constant. For steady flow with free-free boundaries, the critical Rayleigh number has been calculated to be $R a_{c}$ $\approx 657.5 .{ }^{37}$ Using the same values of the parameters as above and $\alpha=1.43 \times 10^{-3} \mathrm{~cm}^{2} / \mathrm{s}$, the minimum temperature difference for unstable flow is $\Delta T_{c}=1.77^{\circ} \mathrm{C}$, larger than the previous estimate but still within reasonable limits.

\section{Measurements of vertical temperature profile}

Direct temperature measurements at the film surface and throughout the water sublayer would clearly establish whether there exists a significant temperature gradient capable of producing a Rayleigh-Bénard like roll in these spreading studies. Unfortunately, for the reasons described here, such temperature profiles proved difficult to measure. We used very thin J-type thermocouples $(0.125 \mathrm{~mm})$ in situ to measure the local temperature during the spreading of a toluene droplet on a quiescent water surface. Since the experimental assembly only allows a few seconds of observation before the spreading film encounters the cell walls, the thermocouples must register the local temperature very quickly for an accurate reading. Even for thin wires of $125 \mu$, the thermocouples can only achieve a $60 \%$ response in 0.04 $\mathrm{s}$ which was not sufficient to produce accurate readings during the rapid spreading studies. In addition, we measured the resolution of the thermocouples to be $\pm 0.25^{\circ} \mathrm{C}$, a value set by the noise level of the multiplex chip in the electronic readout process. The readings achieved during the spreading process were not exactly reproducible and always fell within the temperature range $0{ }^{\circ} \mathrm{C} \leq \Delta T \leqslant 0.25^{\circ} \mathrm{C}$, where $\Delta T$ denotes the temperature difference between the warmer water in the bulk to the cooler water near the surface. Finally, in order to position several thermocouples at a fixed distance from the point of deposition at several depths below the water surface, a small scaffold is required which slows the spreading process. In summary, we suspect that the temperature drop close to the surface is somewhat larger than the noise limit but that custom signal amplification, exceptionally fast response times, and a minimally intrusive scaffold will be required to make these delicate direct measurements. For the time being, the thermal profiles from the sideview shadowgraphs confirm the presence of strong temperature variations in the water sublayer adjacent to the spreading film and the measured values for $\Delta T$ fall in between the estimates from the critical Marangoni and Rayleigh numbers.

\section{CONCLUSION}

Previous studies in the literature have established that the location of the leading edge of a nonvolatile, immiscible surface active film spreading on a deep liquid layer is well described by Eq. (3). The 3/4 spreading exponent reflects the balance between Marangoni driven spreading and viscous retardation from the laminar boundary layer in the liquid support. The spreading speed is controlled by the parameter $S^{1 / 2} /(\mu \rho)^{1 / 4}$. In this paper we have studied the spreading dynamics of volatile immiscible films on a deep water layer for hydrocarbons with different spreading coefficients and vapor pressures. Unlike previous spreading studies for which the location of the advancing front was tracked by surface seeding, we used laser shadowgraphy to detect the location of moving boundaries across the surface of the spreading film. The fastest moving boundary corresponds to the leading edge while the slowest corresponds to the periphery of the droplet reservoir. With this apparatus, we have reproduced the correct spreading law for nonvolatile liquid films for which the spreading exponent is $3 / 4$. Our spreading studies 
with volatile films, however, produce a spreading exponent of $1 / 2$. This exponent is unaffected by using hydrocarbon liquids of different spreading coefficient or vapor pressure. Our studies suggest that Marangoni driven spreading coupled with evaporation displays a common spreading exponent for the leading edge much diminished from the nonvolatile case. We have also shown that while the speed of the leading edge is well correlated with the spreading coefficient, the reservoir speed is better correlated with the vapor pressure. Further work is needed to confirm the dependence of the spreading velocity on these two parameters. The moving boundaries are visualized by the development of regions of high curvature in the spreading film. Shadowgraphy reveals a significant rim associated with the leading edge, which becomes more pronounced in the presence of evaporation. We have initiated an investigation of the temporal evolution of the entire shape of the spreading film using Moiré topography to test recent theoretical predictions of surface elevation created by Marangoni stresses. ${ }^{9}$

We have correlated the surface motion of the spreading film with the development of an unusual thermal boundary layer in the water sublayer. Using sideview shadowgraphy which is sensitive to density variations caused by thermal gradients, we have measured a horizontally flat thermal region capped by a stretched convective roll located right beneath the leading edge of spreading volatile films. Whereas the nonvolatile control sample (silicone oil) showed no fluid circulation, the volatile samples clearly displayed a strong circulation which we attribute to a Rayleigh-Bénard type convective roll. In analogy with this well known instability, the spreading film produces surface cooling due to the evaporation process which is enhanced by the rapid spreading. According to a simple convective-diffusive model of evaporation, the leading edge of the film experiences the largest mass loss and therefore experiences the largest cooling effect. The vertical temperature gradient established at this leading edge is substantial enough to give rise to a convective Rayleigh-Bénard roll which propagates with the leading edge. Comparison with the geometric aspect ratio of classical Rayleigh-Bénard cells reveals that the convective roll in our experiments is stretched in the spreading direction. This stretching is probably due to the fact that the convective roll develops within an overall shear flow created by Marangoni spreading. The significant fluid circulation occurring at the advancing front is expected to produce a well mixed and cooled thermal layer beneath the spreading film. This rapidly advancing, churning horizontal motion produces a thermal layer which is initially of the same approximate thickness as the advancing roll and flat in profile from the leading edge back to the source region.

Estimates based on the available critical Marangoni and Rayleigh numbers in the absence of a shear field produce temperature drops which appear easily achievable in a spreading volatile film. Experiments carefully designed to measure directly the temperature gradient produced in situ during the spreading process would prove very useful. A theoretical model incorporating the rapid horizontal motion due to Marangoni spreading with the superposed rotational motion created by the induced vertical temperature gradient is required in order to determine the new critical values of the Marangoni and Rayleigh numbers appropriate to the geometry corresponding to our experiments. We note, however, that our experiments bear some conceptual similarity to the geometry considered by Unny and Niessenby ${ }^{38}$ in their theoretical work and later investigated experimentally by Koshmieder. ${ }^{37}$ Both studies showed that in the presence of a constant horizontal temperature gradient superimposed onto a vertical one, there develops a net horizontal Marangoni flow superposed on the convective rolls which leads to a lower critical value of the Rayleigh number for buoyancy driven systems. The critical value was found to be as much as six times smaller than the value required in the absence of a horizontal temperature gradient. We suspect that the horizontal motion associated with the spontaneously spreading process can therefore serve to reinforce the usual convective instabilities arising from the presence of a vertical temperature gradient by lowering the critical numbers required for unstable flow.

The presence of a Rayleigh-Bénard instability in these spreading studies may help explain the fact that different volatile liquids spread with the same exponent despite different spreading coefficients and vapor pressures. Its existence provides a large additional source of dissipation which may be responsible for the reduction in spreading exponent from $3 / 4$ to $1 / 2$. We are excited by this possibility and have launched further studies to explore the details of this flow pattern.

\section{ACKNOWLEDGMENTS}

A.D.D. gratefully acknowledges support from the Ministère Francais de la Recherche postdoctoral fellowship program. S.M.T. would like to thank the National Science Foundation for financial support through a Research Initiation and CAREER award (S.M.T.) as well as a MRSEC seed grant distributed through the Princeton Materials Institute (S.M.T.). Grants from the Exxon Education Foundation and the Can Manufacturers Institute were used to purchase optical equipment for these studies.

${ }^{1}$ J. A. Fay, "The spread of oil slicks on a calm sea" in Oil on the Sea, edited by D. Hoult (Plenum, New York, 1969).

${ }^{2}$ D. Hoult, "Oil spreading on the sea," Annu. Rev. Fluid Mech. 4, 341 (1972).

${ }^{3}$ C. Huh, M. Inoue, and S. G. Mason, "Unidirectional spreading of one liquid on the surface of another," Can. J. Chem. Eng. 53, 367 (1975).

${ }^{4}$ N. D. DiPietro, C. Huh, and R. Cox, "The hydrodynamics of the spreading of one liquid on the surface of another," J. Fluid Mech. 84, 529 (1978).

${ }^{5}$ M. Foda and R. G. Cox, "The spreading of thin liquid films on a water-air interface," J. Fluid Mech. 101, 33 (1980).

${ }^{6} \mathrm{Z}$. Dagan, "Spreading of films of adsorption on a liquid surface," Physico Chem. Hydrodyn. 5, 43 (1984).

${ }^{7}$ D. W. Camp and J. C. Berg, "The spreading of oil on water in the surface tension regime," J. Fluid Mech. 184, 445 (1987).

${ }^{8}$ J. F. Harper, "The leading edge of an oil slick, soap film, or bubble stagnant cap in stokes flow," J. Fluid Mech. 237, 23 (1992).

${ }^{9} \mathrm{O}$. E. Jensen, "The spreading of insoluble surfactant at the free surface of a deep fluid layer," J. Fluid Mech. 293, 349 (1995).

${ }^{10}$ A. W. Adamson, Physical Chemistry of Surfaces (Wiley, New York, 1990).

${ }^{11}$ A. Gericke, J. Simon-Kutscher, and H. Huhnerfuss, "Influence of the 
spreading solvent on the properties of monolayers at the air/water interface,' Langmuir 9, 2119 (1993).

${ }^{12}$ W. D. Harkins, The Physical Chemistry of Surface Films (Reinhold, New York, 1952)

${ }^{13}$ D. W. Camp, Ph.D. thesis, University of Washington, Dept. of Chem. Eng., 1985.

${ }^{14}$ P. Joos and J. van Hunsel, "Spreading of aqueous surfactant solutions on organic liquids,', J. Colloid Interface Sci. 106, 161 (1985).

${ }^{15}$ J. T. Davies and E. Rideal, Interfacial Phenomena (Academic, New York, 1963), p. 26.

${ }^{16}$ M. Santiago, M. Vignes-Adler, and M. G. Velarde, in Fluid Physics, Lecture Notes of Summer Schools, Series B, Vol. 5, edited by M. G. Velarde and C. I. Christov (World Scientific, Singapore, 1995), p. 275.

${ }^{17}$ M. Santiago-Lopez, Ph.D. thesis, Universitad Complutense de Madrid, Facultad de Ciencias Fisicas, 1995.

${ }^{18}$ P. Pomerantz, W. C. Clinton, and W. A. Zisman, "'Spreading pressure and coefficients, interfacial tensions, and adhesion energies of the lower alkanes, alkenes, and alkyl benzenes on water,' J. Colloid Interface Sci. 24, 16 (1967).

${ }^{19}$ G. W. Hannaway and Associates, Wyndham Hannaway Imaging System, Boulder, CO, 1984.

${ }^{20}$ R. Goldstein, Fluid Mechanics Measurements (Hemisphere, New York, 1983), p. 394.

${ }^{21}$ R. Sellin, "Existence of a surface tension discontinuity at a liquid free surface," Nature (London) 217, 536 (1968).

${ }^{22} \mathrm{C}$. W. McCutchen, "Surface films compacted by moving water: demarcation lines reveal film edges,', Science 170, 61 (1970).

${ }^{23}$ R. S. McDowell and C. W. McCutchen, "The thoreau-reynolds ridge, a lost and found phenomena,' Science 172, 973 (1971).

${ }^{24}$ J. C. Scott, "Flow beneath a stagnant film on water: the reynolds ridge,", J. Fluid Mech. 116, 283 (1982).

${ }^{25}$ A. Warncke, M. Gharib, and T. Roesgen, "Flow measurements near a reynolds ridge,' J. Fluids Eng. 118, 621 (1996).

${ }^{26}$ J. F. Harper and J. N. Dixon, "The leading edge of a surface film on contaminated flowing water,' Proc. Fifth Australasian Conf. Hydraulics Fluid Mech. 2, 499 (1974).

${ }^{27}$ E. Landt and M. Volmer, Z. Phys. Chem. (Munich) 122, 398 (1926).
${ }^{28}$ W. D. Garrett and W. R. Barger, "Factors affecting the use of monomolecular surface films to control oil pollution on water," Environ. Sci. Technol. 4, 123 (1970).

${ }^{29}$ S. M. Troian and J. M. Drake, "Pattern generation in the dewetting of volatile films from the surface of a thick water support," (unpublished).

${ }^{30}$ R. N. O'Brien, A. I. Feher, and J. Leja, "Interferometric and hydrodynamic flow profiles produced in water by a spreading monolayer,' J. Colloid Interface Sci. 51, 366 (1975).

${ }^{31}$ A. D. Dussaud, S. M. Troian, and S. R. Harris, "Fluorescence visualization of a convective instability which modulates the spreading of volatile films,", submitted to Phys. Fluids (1997).

${ }^{32}$ W. Lempert et al., "Flow tagging velocimetry in incompressible flow using photo-activated nonintrusive tracking of molecular motion," Exp. Fluids 18, 249 (1995).

${ }^{33}$ Camp and Berg (Ref. 7) measured the thickness and velocity profiles of several spreading oils and from this data deduced the surface concentration. For a typical oil like oleic acid, they found that in the forward part of the film where the spreading velocity is constant and the film behaves as a rigid plate, the surface concentration was practically constant, varying slightly from $4 \times 10^{-10} \mathrm{~mol} / \mathrm{cm}^{2}$ at the leading edge to $6 \times 10^{-10} \mathrm{~mol} / \mathrm{cm}^{2}$ at the trailing edge. Beyond this point there was a large jump in velocity (the "acceleration zone") and a subsequent increase in the surface concentration. From their studies, the forward part of the film which behaves as a rigid plate can be approximated as a region of constant concentration.

${ }^{34}$ R. B. Bird, W. E. Stewart, and E. N. Lightfoot, Transport Phenomena (Wiley, New York, 1960).

${ }^{35}$ J. R. A. Pearson, "On convection cells induced by surface tension,' J. Fluid Mech. 4, 489 (1958).

${ }^{36}$ L. E. Scriven and C. V. Sternling, "On cellular convection driven by surface tension gradients: effects of mean surface tension and surface viscosity,', J. Fluid Mech. 19, 321 (1964).

${ }^{37}$ E. L. Koschmieder, 'Bénard convection,' Adv. Chem. Phys. 26, 177 (1974).

${ }^{38}$ T. Unny and P. Niessen, "Thermal instability in fluid layers in the presence of horizontal and vertical temperature gradients,' J. Appl. Mech. 36, 121 (1969). 